A. G. Constantin, H. Wang, J. C. Martinez-Trujillo and J. D. Crawford

J Neurophysiol 98:696-709, 2007. First published Jun 6, 2007; doi:10.1152/jn.00206.2007

You might find this additional information useful...

Supplemental material for this article can be found at:

http://jn.physiology.org/cgi/content/full/00206.2007/DC1

This article cites 103 articles, 51 of which you can access free at:

http://jn.physiology.org/cgi/content/full/98/2/696\#BIBL

Updated information and services including high-resolution figures, can be found at:

http://jn.physiology.org/cgi/content/full/98/2/696

Additional material and information about Journal of Neurophysiology can be found at:

http://www.the-aps.org/publications/jn

This information is current as of September 4, 2007. 


\title{
Frames of Reference for Gaze Saccades Evoked During Stimulation of Lateral Intraparietal Cortex
}

\author{
A. G. Constantin, ${ }^{1,2,3}$ H. Wang, ${ }^{1,2}$ J. C. Martinez-Trujillo, ${ }^{1,5}$ and J. D. Crawford ${ }^{1,2,3,4}$ \\ ${ }^{1}$ Centre for Vision Research, ${ }^{2}$ Canadian Institute of Health Research Group for Action and Perception, and ${ }^{3}$ Department of Biology, \\ York University, Toronto, Ontario; ${ }^{4}$ Departments of Psychology and Kinesiology and Health Sciences, York University, Toronto, Ontario, \\ Canada, M3J 1P3; and ${ }^{5}$ Department of Physiology, McGill University, Montreal, Quebec, Canada
}

Submitted 26 February 2007; accepted in final form 25 May 2007

\begin{abstract}
Constantin AG, Wang H, Martinez-Trujillo JC, Crawford JD. Frames of reference for gaze saccades evoked during stimulation of lateral intraparietal cortex. J Neurophysiol 98: 696-709, 2007. First published June 6, 2007; doi:10.1152/jn.00206.2007. Previous studies suggest that stimulation of lateral intraparietal cortex (LIP) evokes saccadic eye movements toward eye- or head-fixed goals, whereas most single-unit studies suggest that LIP uses an eye-fixed frame with eye-position modulations. The goal of our study was to determine the reference frame for gaze shifts evoked during LIP stimulation in head-unrestrained monkeys. Two macaques (M1 and M2) were implanted with recording chambers over the right intraparietal sulcus and with search coils for recording three-dimensional eye and head movements. The LIP region was microstimulated using pulse trains of $300 \mathrm{~Hz}, 100-150 \mu \mathrm{A}$, and $200 \mathrm{~ms}$. Eighty-five putative LIP sites in $M 1$ and 194 putative sites in $M 2$ were used in our quantitative analysis throughout this study. Average amplitude of the stimulation-evoked gaze shifts was $8.67^{\circ}$ for $M 1$ and $7.97^{\circ}$ for $M 2$ with very small head movements. When these gaze-shift trajectories were rotated into three coordinate frames (eye, head, and body), gaze endpoint distribution for all sites was most convergent to a common point when plotted in eye coordinates. Across all sites, the eye-centered model provided a significantly better fit compared with the head, body, or fixed-vector models (where the latter model signifies no modulation of the gaze trajectory as a function of initial gaze position). Moreover, the probability of evoking a gaze shift from any one particular position was modulated by the current gaze direction (independent of saccade direction). These results provide causal evidence that the motor commands from LIP encode gaze command in eye-fixed coordinates but are also subtly modulated by initial gaze position.
\end{abstract}

\section{N T R O D U C T I O N}

To obtain high-acuity vision, one needs to rotate the eyefixed fovea toward space-fixed targets using rapid "gaze shifts." Usually, gaze shifts are associated with both rapid eye movements called saccades and somewhat slower movements of the head in roughly the same direction (Bizzi et al. 1976; Guitton and Volle 1987). To accurately program these movements, the brain must transform the information from coordinate systems centered on different input sensory organs (the eyes, the skin, the ear) into the head and body-fixed frames preset to the muscles that control the eyes and head (Andersen et al. 1997; Cohen and Andersen 2002; Colby 1998; Graziano and Gross 1998; Soechting and Flanders 1992). An important question in gaze control is what are the intermediate frames of reference used by the cortex to represent gaze targets?

Address for reprint requests and other correspondence: J. D. Crawford, Center for Vision Research, York University, 4700 Keele St., Toronto, Ontario M3J 1P3, Canada (E-mail: jdc@yorku.ca).
The main cortical centers involved in saccade control include the frontal eye fields (FEFs) (Astafiev et al. 2003; Hanes et al. 1998; Russo and Bruce 1996; Schall 2002; Sparks et al. 2001; Wurtz et al. 2001), the supplementary eye fields (SEFs) (Chen and Walton 2005; Martinez-Trujillo et al. 2003a,b, 2004), and the lateral intraparietal area (LIP) (Goldberg et al. 2002; Pare and Wurtz 1997, 2001; Thier and Andersen 1996, 1998). Neurophysiological studies suggest that FEF encodes information in an eye-fixed (retinotopic) frame of reference (Bruce et al. 1985; Russo and Bruce 1993; Tu and Keating 2000), whereas both unit recording and microstimulation studies suggest that SEF uses multiple reference frames (Dominey et al. 1997; Martinez-Trujillo et al. 2004; Park et al. 2006; Russo and Bruce 1996, 2000; Tehovnik et al. 2000).

In the present study, we focused on the question of what reference frame does LIP use to encode gaze. Previous singleunit recording studies have suggested that LIP might encode saccades using eye (Andersen and Buneo 2002; Boussaoud and Bremmer 1999; Colby and Duhamel 1996; O'dhaniel et al. 2005), head (O'dhaniel et al. 2005), body/space (Brotchie et al. 1995; Snyder et al. 1998), or a more complex reference frame (Platt and Glimcher 1997). A number of studies suggested that LIP neurons have a retinotopic (eye-fixed) motor field, but its activity is modulated by eye/head position (Andersen et al. 1985; Barash et al. 1991; Li and Andersen 2001; Linden et al. 1999; Mazzoni et al. 1996). This suggests that although these neurons may code target locations in eye-fixed coordinates, they also possess the information necessary to transform this into other frames (Andersen 1989; Andersen and Zipser 1988). Electrical microstimulation studies (Kurylo and Skavenski 1991; Shibutani et al. 1984; Thier and Andersen 1996) have reported stimulation-evoked saccades with goals that were best defined in an eye- or head-fixed reference frame, depending on the site.

All these previous studies had the limitation that they restricted the head movements of their subjects, either completely or by limiting the head to one axis of rotation (Brotchie et al. 1995; Thier and Andersen 1998). However, previous microstimulation studies of other brain areas (SC, SEF) involved in gaze motor control have showed that when the head is free to move naturally, the head contribution to the overall gaze shifts influences the amplitude of the stimulation-evoked gaze shifts (Freedman et al. 1996; Martinez-Trujillo et al. 2003a; Roucoux et al. 1980). Moreover, the eyes and the head

\footnotetext{
The costs of publication of this article were defrayed in part by the payment of page charges. The article must therefore be hereby marked "advertisement" in accordance with 18 U.S.C. Section 1734 solely to indicate this fact.
} 
move in a three-dimensional (3-D) space performing movements about the corresponding 3-D, i.e., vertical, horizontal, and torsional (line of sight) axes (Glenn and Vilis 1992; Tweed et al. 1995). Therefore it is important to measure these three components when we record the eye and head movements during head-unrestrained gaze shifts (Klier and Crawford 2003; Klier et al. 2003). Thus far, no electrophysiological study of macaque area LIP has used monkeys the heads of which were completely unrestrained (i.e., allowed to move freely both the eyes and the head) and measured eye and head movements in 3-D. These procedures - recording 3-D rotations in head-unrestrained monkeys-are essential for discriminating movement coding in different reference frames (Klier et al. 2002; Soechting and Flanders 1992).

The goal of this study was to analyze 3-D gaze saccades evoked during electrical stimulation of LIP in head-unrestrained macaques. One possibility is that stimulating the LIP will evoke fixed-vector like gaze shifts_-gaze movements with constant amplitude and direction, which vary as a function of the stimulation location (Hanks et al. 2006; Kurylo and Skavenski 1991; Schiller and Tehovnik 2005; Thier and Andersen 1996). A second possibility is that stimulation of LIP will produce gaze shifts directions that are modulated by initial eye position, consistent with a retina-fixed (eye-fixed) pattern (Crawford and Guitton 1997a; Klier et al. 2001). Another possibility is that LIP stimulation will evoke gaze shifts toward a goal that is fixed with respect to the head or with respect to space (Cutrell and Marrocco 2002; Mushiake et al. 1999; Shibutani et al. 1984; Thier and Andersen 1998).

Based on a recent study of the superior colliculus (SC) (Klier et al. 2001), on the information that LIP is functionally situated upstream from the SC (Ferraina et al. 2002; Gaymard et al. 2003; Pare and Wurtz 1997; Wurtz et al. 2001), and on previous single-unit studies (Andersen et al. 1990a; Barash et al. 1991; Colby et al. 1995, 1996; Ipata et al. 2006; Mazzoni et al. 1996), we hypothesized that LIP uses an eye-centric reference frame to code for the eye movements while the head is free to move. However, based on the numerous studies that have shown eye-position "gain field" modulations in LIP (i.e., where location of the receptive field remains fixed in eye coordinates, but the sensitivity gain of the neuron is increased or decreased as a linear function of eye position) (Andersen et al. 1985, 1990b), we also analyzed the data to detect if the evoked movements were also modulated by gaze position in the same way. We found that stimulation of LIP in headunrestrained monkeys did produce an eye-fixed pattern of gaze shifts but that the probability of evoking these gaze shifts depended on initial gaze position in a way that may be related to the existence of eye-position gain fields.

\section{METHODS}

\section{Animal preparation}

The surgical and experimental procedures used in this study were approved by the York University Animal Care Committee and were in compliance with the Canadian Council on Animal Care policy on the use of laboratory animals. Two female monkeys, Macaca mulata (rhesus), underwent surgery under general anesthesia (isoflurane, $1.5 \%$; and ketamine, $10 \mathrm{mg} / \mathrm{kg}$ ), during which a head post for immobilizing the head, a plastic recording chamber (Narishige), and sockets for cable connection (located dorsally) were implanted into each animal; all fixed to a dental acrylic cap attached to the skull by orthopedic screws (Synthes). The recording chamber of $20 \mathrm{~mm}$ diam was positioned on the skull at $5 \mathrm{~mm}$ posterior and $12 \mathrm{~mm}$ lateral in stereotaxic coordinates, over the right lateral intraparietal sulcus (IPS; Fig. 1A). The head post, which consisted of a stainless steel cylinder with a rapid release mechanism (for details, see Crawford et al. 1999), was located frontally. We also implanted two custom-built scleral search coils (copper wire), of $5 \mathrm{~mm}$ diam, fitted subconjunctivally in the nasal quadrants of one eye. This allowed 3-D measurements of eye orientation (for details, see Tweed and Vilis 1990; Tweed et al. 1990). After the surgery, the monkeys were allowed $2 \mathrm{wk}$ for recovery before initiating the training and experimenting sessions.

During the experiments, each animal wore a primate jacket and was trained to sit upright in a modified Crist primate chair (Crist Instruments), which constrained the movements of the torso but still allowed for natural and unrestricted movements of the eyes and the head, except that vision was obstructed toward directions $>50^{\circ}$ downward (for details, see Constantin et al. 2004a). The primate chair was placed near the center $( \pm 15 \mathrm{~cm})$ of three orthogonal magnetic fields (Tweed et al. 1990), and coil signals were recorded at a sampling frequency of $1,000 \mathrm{~Hz}$. During experimentation, we temporarily fitted the acrylic cap of the animal with two orthogonal coils, of 1-cm diam, for 3-D measurements of head orientation. Electrode penetrations were made using a small hydraulic microdrive (Narishige model MO-95S) customized to fit onto a stage with a radial placement system, positioned on the top of the recording chamber. The weight of this entire system was $48 \mathrm{~g}$, which remained constant throughout experimentation.

\section{Training and experiments}

Each animal was trained only with the head unrestrained (headfree); they were trained to perform head-free gaze shifts to lightemitting diodes (LEDs) in the dark. The LED screen was situated at a distance of $80 \mathrm{~cm}$ in front of the monkey. A total of 17 LEDs located at \pm 20 and $\pm 40^{\circ}$, on vertical, horizontal, and oblique axes, were positioned on the screen. The animals were cued to orient their gaze toward a specific quadrant - upper left or right, lower left or right(with the help of a short, high pitched sound) and were then required to fixate an LED illuminated in that quadrant. The LED was then turned off, but animals were trained to maintain the gaze on the target LED position for a period of $500-800 \mathrm{~ms}$, within a fixation window of 5 to $10^{\circ}$ centered on the LED, to receive a juice reward.

The electrophysiology experiments began after a training period of $3 \mathrm{wk}$, in both monkeys. Single-unit recording and microstimulation sessions were performed with the use of tungsten microelectrodes $(0.15 \sim 2.5 \mathrm{~m} \Omega$ impedance at $1 \mathrm{kHz}, \mathrm{FHC})$ directed through a stainless steel guide tube, which penetrated $2-3 \mathrm{~mm}$ beyond the dura. During each session, one to two electrode penetrations (tracks) were made. During each track, the electrode was slowly lowered while restraining the head of the animal for a short interval, and single-unit activity was monitored using an oscilloscope and an audio monitor and compared on-line to the visually guided gaze, eye, and head movements. When correlations between a neuron's activity and eye movements were detected, we stimulated the corresponding site using $10-20$ cathodal trains $(100-300 \mathrm{~Hz}, 80-100 \mu \mathrm{A}$, and $200 \mathrm{~ms})$. If the electrical microstimulation evoked eye movements, we allowed the monkey's head to go free and commenced the experiments.

We found that higher stimulation currents were required to evoke movements with the head free $(300 \mathrm{~Hz}, 100-150 \mu \mathrm{A}, 200 \mathrm{~ms})$. It is possible that these higher stimulation thresholds were required to overcome greater activation of the gaze fixation system, which animals might engage to overcome the greater inertia of the head-free system (Corneil et al. 2002). These current intensities were similar with those used in previous studies (Thier and Andersen 1996, 1998) that stimulated LIP using mostly currents of 100-200 $\mu \mathrm{A}$. We delivered stimulation for each target position, in a random order. 

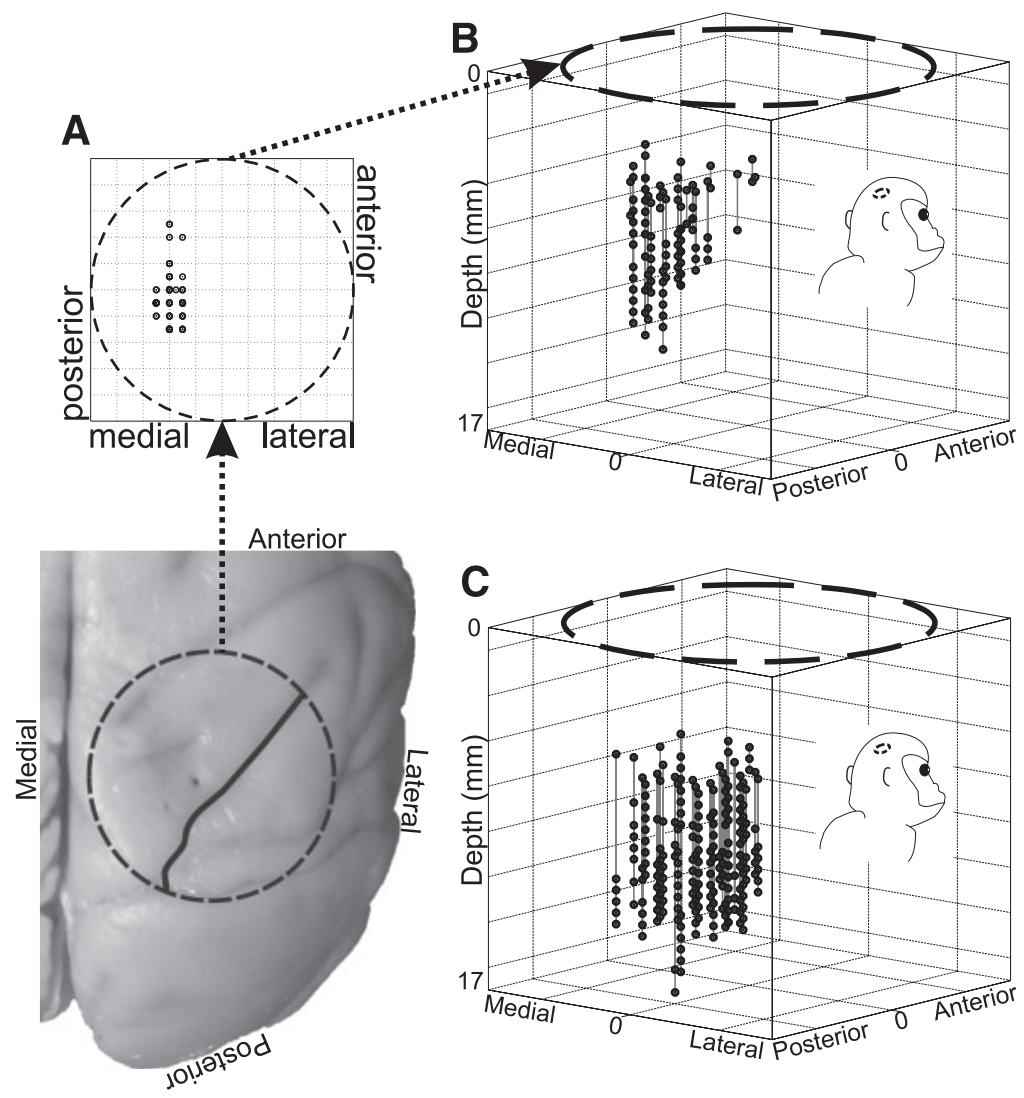

FIG. 1. Lateral intraparietal area (LIP) map. A: top view of the right brain hemisphere in $M 1$. The dashed circle indicates the recording chamber location. The thick black line accentuates the intraparietal sulcus. Guide tubes penetrated medially to IPS and then electrodes passed vertically through the sulcus to the lateral intraparietal area (LIP). Top: site location onto the 2-dimensional (2-D) chamber coordinates. (anterior-posterior versus medial-lateral axis), top view. $B$ : 3-D reconstituted map with the stimulated sites location (black circle), with a total of 85 stimulation sites from M1. C: 3-D map with a total of 194 sites (black circle) from M2. The little monkey head caricatures allow for a better orientation of the cube position.
Stimuli were delivered 400-800 ms after the LED was extinguished while the animal fixated in complete darkness. Thus the animals received the juice reward immediately after stimulation offset. We applied the electrical stimulus for an average of 50 times per site with a range of 30-80 (depending on the probability of evoking a movement from a given site). The order of illumination of the LEDs was randomized; in most cases, this resulted in $\geq 2$ stimulations per target and maximum of 10. Afterward we advanced the electrode $0.5 \mathrm{~mm}$ vertically and repeated the process. The area was thoroughly investigated with tracks positioned $1 \mathrm{~mm}$ apart (plotted as gray, parallel lines in Fig. 1, $B$ and $C$ ), until we had fully explored the areas that did evoke movements and reached areas that did not evoke any movements. Following these procedures, movements evoked from 141 sites (from 47 electrode penetrations) in monkey $1(M 1)$ and 214 sites (from 47 electrode penetrations) in monkey 2 (M2) were analyzed. The subsequent data analysis reduced the number of sites used in this study to 85 sites in $M 1$ and 194 sites in $M 2$.

\section{Data analysis}

Data analysis was conducted off-line using a personal computer (PC) and a custom-made program. We used the computer to convert the coil signals into position signals in quaternions, for the eyes in space (gaze) and head in space (head), and to calculate the orientation for the eyes relative to head (eye) (Crawford et al. 1999; Tweed et al. 1990). Quaternions represent positions as a fixed-axis rotation from a reference position, i.e., when the animal is looking straight ahead (Crawford et al. 1999; Tweed et al. 1990; Westheimer 1957). From these quaternions, we computed the gaze trajectories, angles of rotation, and angular velocities (Crawford et al. 1999; Tweed et al. 1990).

The beginning and the end of stimulation-evoked gaze, eye, and head movements were manually selected, i.e., by visually inspecting position and velocity components plotted as a function of time and then placing a vertical cursor at the appropriate onset/offset points.
Peak velocities for the selected trajectories were then computed. We included movements with maximum gaze velocities $>50 \%$ and latencies (time from stimulation onset to peak velocity) $<200 \mathrm{~ms}$ (the duration of the electrical stimulus). In addition, we plotted the distribution of all movement latencies and excluded movements with values larger than the mean plus 2 SD. Additionally, colliding saccades (saccades resulting from microstimulation delivered during a visually evoked saccades) (Schlag and Schlag-Rey 1990) were removed from the data set. In general, stimulation did not evoke saccades on every trial, even at sites where it did sometimes evoke movements. We only quantified data from sites in which we evoked at least seven movements from at least three of the four visual quadrants (i.e., minimum 2 evoked movements from each quadrant), which resulted in 85 sites in $M 1$ and 194 sites in $M 2$.

During our experiments, we observed that the probability of evoking a saccade during stimulation depended on gaze position. Moreover the distribution of evoked movements as a function of initial gaze orientation varied from site to site. We quantified the probability of evoking saccades as a function of initial eye orientation by calculating a probability vector (PV) for each site. Details of our method are provided in the results section. For this PV analysis, we only selected the sites in which we applied minimum two stimulations in no less than three quadrants, with initial gaze orientation that covered an area of at least $\pm 20^{\circ}$ in each of the three quadrants. This resulted in 28 (of 85) sites for $M 1$ and 180 (of 194) sites for M2; only these sites were used in the PV analysis.

We used two methods to assess the reference frame that best characterizes the goal of the stimulation-evoked movements. To characterize the typical results from the stimulation of each site, we used a method described in Klier et al. (2001) and Martinez-Trujillo et al. (2004). First we calculated a characteristic vector (CV) for stimulation-evoked gaze shifts from each site. This vector represents the trajectory that would be expected from stimulating the site when the eyes and head of the animal are pointed straight ahead at the 
beginning of the stimulation. Second, we used initial eye and head orientation quaternions to rotate gaze trajectories into different coordinate systems (reference frames) - from eye-in-space to head and to eye-in-head reference frames (Martinez-Trujillo et al. 2004).

To characterize how convergent the gaze trajectories are in different reference frames, we used different methods. First, we fitted an ellipse to the endpoints of the gaze movements using the least-squares method, such that $95 \%$ of the endpoints fall inside the ellipse (Martinez-Trujillo et al. 2004). Second, we computed the convergence of the gaze trajectories as a function of the initial gaze position resulting in the computation of the convergence index (CI) for the movement direction (on-axis component, i.e., for the component parallel to the characteristic gaze vector) and for the movement amplitude (off-axis component, i.e., for the component orthogonal to the characteristic gaze vector) (Klier et al. 2001; Martinez-Trujillo et al. 2003a, 2004). Finally, we compared such indices with the ones predicted by models of different reference frames coding by computing the normalized residuals for each model, which allowed us to compare the data plotted in the different reference frames (Martinez-Trujillo et al. 2004). The latter analysis accounted for the possibility that the data may not neatly fit into one reference frame or another, i.e., if the endpoints of the stimulation-evoked movements did not converge perfectly in any frame.

\section{Anatomical reconstruction of the stimulated area}

The first step in the reconstruction of the stimulation sites was made by assembling a 3-D map of the recording chamber with anteriorposterior, lateral-medial, and depth coordinates and superimposing the coordinates of the stimulation sites onto this map. Figure 1 shows the location of the 85 LIP sites (black circle) for $M 1$ (Fig. 1, $A$ and $B$ ) and 194 LIP sites for $M 2$ (Fig. 1C). Each of these sites met the analysis criteria described in Data analysis.

The second step in the anatomical reconstruction consists in histological analysis of the stimulated brain areas. While $M 1$ was anesthetized, LIP sites chosen as points of reference were marked using controlled lesions (1.5-mA currents were delivered for $15 \mathrm{~s}$ ). Next, $M 1$ was killed, and its brain was perfused with phosphate-buffered paraformaldehyde. High-resolution digital images of the brain were taken before sectioning (Fig. 1A). The tissues containing the stimulation sites were then sectioned on a freezing microtome at $100 \mu \mathrm{m}$. Visual inspection confirmed that the stimulation sites reported in this study were situated on the lateral bank of the intraparietal sulcus (IPS). M2 is still being actively used in other experiments but step 1 (stereotaxic mapping) showed similar results as in $M 1$ (Fig. 1C).

\section{RES ULTS}

\section{Stimulation-evoked gaze movements: general observations}

Putative LIP sites evoked leftward gaze shifts with a large range of vertical movement components and with amplitudes varying between 1.66 and $23.87^{\circ}$ in $M 1$ and between 1.9 and $22.77^{\circ}$ in $M 2$. Figure 2, $A$ and $C$ plots horizontal components as a function of time for one typical stimulation-evoked movement. Figure $2 A$ shows the horizontal components of gaze (thick line), head (thin line) and eye (dotted line) position, and the corresponding velocity traces (Fig. 2C). In this example (as in most of our data), the gaze shift was accomplished almost completely by an eye movement, accompanied by an insignificant head component. Figure $2 D$ shows the main sequence plots (peak velocity as a function of amplitude) for all evoked movements from $M 1$ (black circle) and $M 2$ (white circle), divided into bins and averaged. There was no statistical difference between the data from the two animals $(P=0.89$, Student's $t$-test). The latency distribution for the stimulation-
A
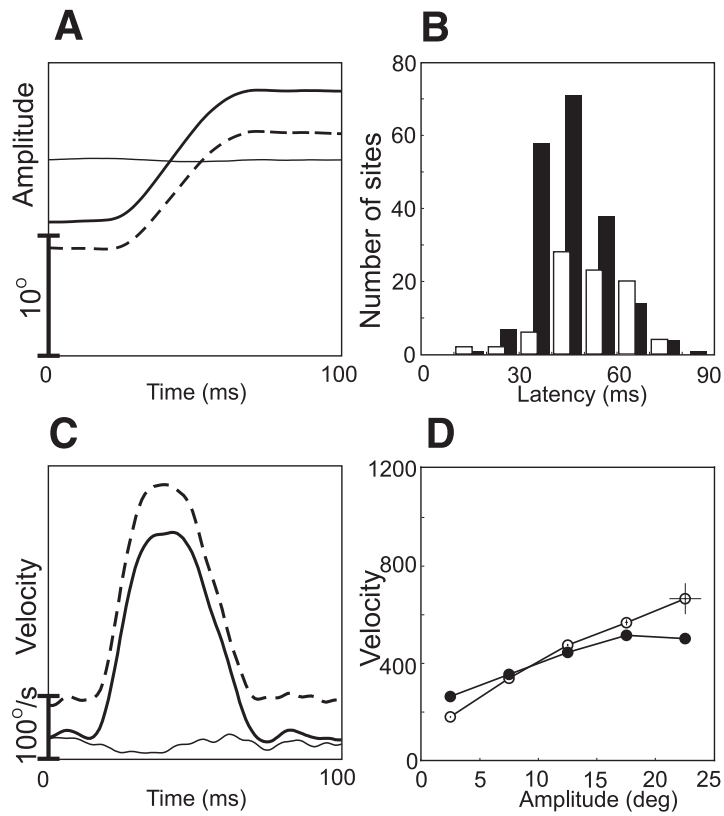

FIG. 2. A: horizontal position as a function of time for a typical stimulationevoked gaze shift (thick line) and the corresponding head trace (thin line) and eye trace (dotted line). $C$ : horizontal velocity as a function of time corresponding to the position traces in $A$; plotted for gaze (thick line), head (thin line), and eye (dotted line). $B$ : latency distribution per site for the stimulation-evoked gaze shifts, showing the averages of $10^{\circ}$ bins, for $M 1$ (black) and $M 2$ (white) columns. $D$ : main sequence plots for all the stimulation-evoked gaze-shifts, using $10^{\circ}$ bins, plotted for $M 1$ (black circle) and M2 (white circle), with no statistical difference between the 2 data sets.

evoked gaze shifts for the two monkeys is plotted in Fig. 2B, showing a peak between 40 and $60 \mathrm{~ms}$ in both animals.

Figure 3 plots 2-D movement traces evoked by stimulating three LIP sites, showing one site with an upward gaze component (left) and two sites with a downward gaze component (middle and right). Each line illustrates the 2-D trajectory of a stimulation-evoked movement, starting from a different initial gaze position. The small circles $(\bigcirc)$ point to the end of the movements. We plotted the trajectories for gaze (Fig. 3, $A-C$ ), head $(D-F)$, and eye-in-head $(G-I)$ movements evoked by stimulating three different LIP sites. As stated in METHODS, stimulation was applied $\leq 10$ times per target location. For example, for the central target, stimulation evoked either three (sites 1 and 3 ) or eight movements (site 2). The movements evoked from each target position had similar direction and amplitude, as in fact did movements from all of the different target positions.

For the illustrated sites, the stimulation-evoked gaze shifts were almost parallel in direction and had similar amplitudes for each trial (Fig. 3, $A-C$ ) with predominantly leftward eye/ saccade-movement components (Fig. 3, D-F) and very small head-movement components (Fig. 3, $G-I$ ). Note that despite this lack of head movement during stimulation, different initial gaze positions (Fig. 3, $A-C$ ) were associated with a considerable amount of variability in head orientation simply due to the natural behavior of the animal (Fig. 3, $D-F$ ). This variability was required for our reference frame analysis (see following text). For such analysis, we will use the data shown in the top panels, i.e., gaze movements. The details of eye-head coordination will be dealt with elsewhere (Constantin et al. 2004b). 

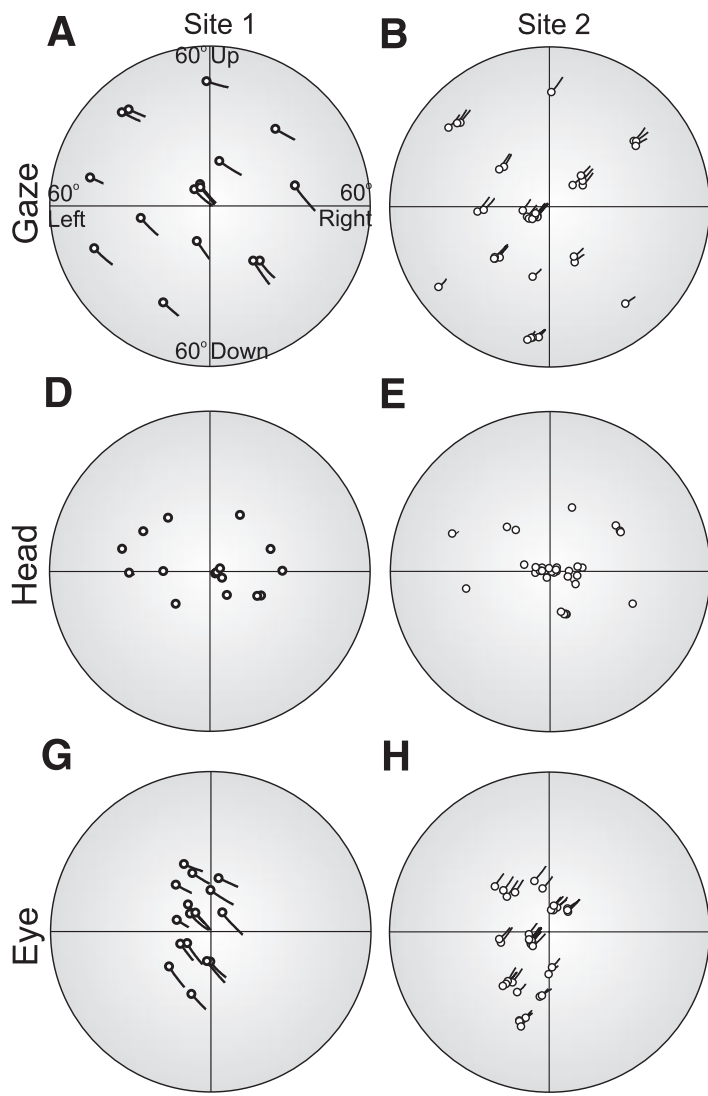

E

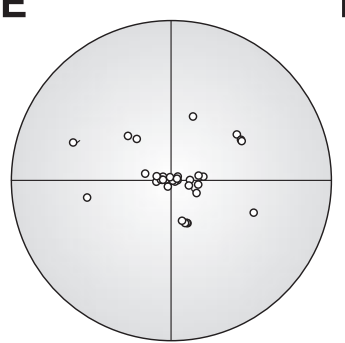

$\mathbf{H}$
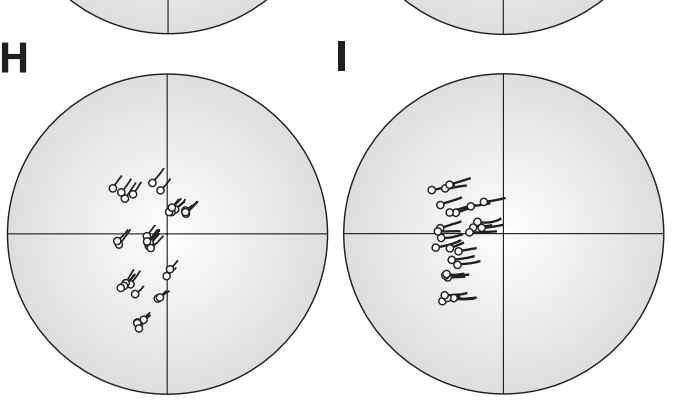

I

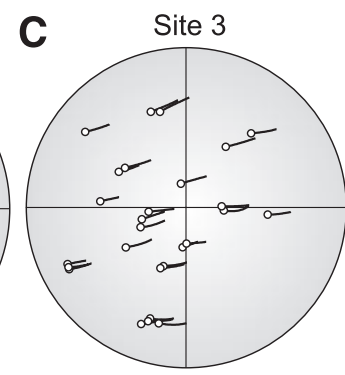

$\mathbf{F}$

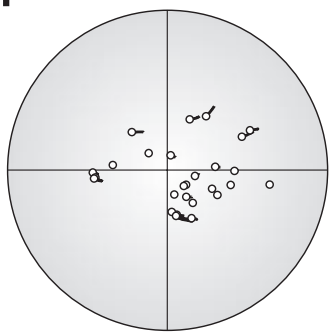

FIG. 3. Behind view of the 2-D trajectories of gaze $(A-C)$, head $(D-F)$, and eye saccade $(G-I)$ evoked by stimulating 3 putative LIP sites, site 1 (left), site 2 (middle), and site 3 (right). Stimulation evoked $\leq 10$ movements per target location. Each point corresponds with the tip of the unit-length vector (computed from quaternions - see METHODS) and represents the position in space for gaze, head, and eye in head with the horizontal and vertical components projected from behind, plotted on a linear scale. $\bigcirc$, end of the movement. The coordinate scale is linear. The sites evoked leftward gaze movements with similar amplitudes but with an upward component $(A)$ or a downward component $(B$ and $C$ ). All sites evoked very small head movements, averaged at $<1^{\circ}$ in amplitude $(D-F)$. The eye-saccade components of the gaze $(G-I)$ were similar in amplitude and direction with the stimulation-evoked gaze shifts, in both sites.
Each stimulation site evoked a different size and direction of gaze shift. To document the typical results of each stimulation site and to compare the evoked gaze shifts across sites, we calculated the $\mathrm{CV}$, using only the stimulation-evoked movements that met the criteria stipulated in METHODs. Each CV indicates the theoretical movement trajectory evoked by stimulating a given site when the gaze is pointing straight ahead.

Figure 4 ( $A$ and $B$ ) illustrates the distribution of the CVs for all sites, from $M 1$ (Fig. 4A) and $M 2$ (Fig. 4B); each arrow represents the CV for one particular site. The purpose of this plot and the following analyses is to consider the amplitudes and directions of the stimulus-evoked gaze shifts as a popula-
A

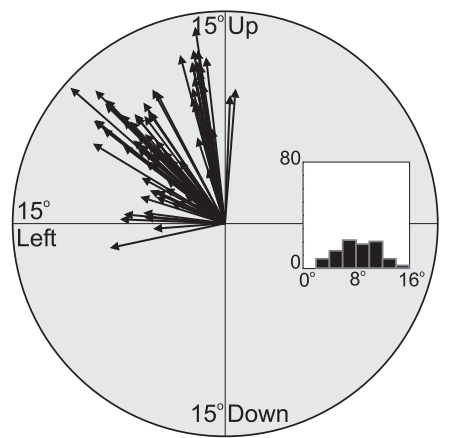

B

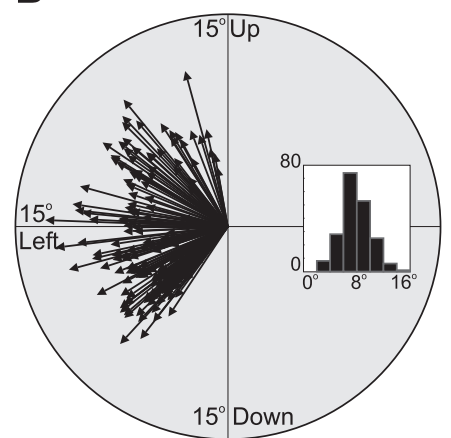

FIG. 4. Characteristic vectors (CVs) for gaze. $A$ and $B$ : stimulating LIP evoked leftward gaze shifts with up- and downward components; the amplitude and direction of the CV were similar between the 2 monkeys. $\rightarrow, \mathrm{CV}$ computed for 1 particular site. The LIP area for $M 2(B)$ was more thoroughly investigated; hence the increased number of downward stimulation-evoked movements. Insets: distribution the $\mathrm{CV}$ amplitudes for each corresponding monkey. tion. For $M 1$ (Fig. 4A), the amplitude of the CVs varied between 2.65 and $14.59^{\circ}$ with an average of $8.36 \pm 2.90^{\circ}$. The CV's direction was mostly toward the upper-left quadrant of space. For $M 2$ (Fig. 4B), the amplitude of the CVs varied between 2.1 and $14.12^{\circ}$ with an average of $7.71 \pm 2.25^{\circ}$. For both monkeys, the calculated CV's direction was always contralateral to the location of the stimulated area, i.e., stimulation of right LIP evoked leftward movements with a wide range of vertical directions, more evident in $M 2$ where the CV's direction spread out evenly between the upper and lower left quadrant; in $M 1$, the CV directions did not spread evenly with just two sites with downward movements. The embedded histograms show the distribution of the CV's amplitudes for each monkey; these distributions were relatively similar for both monkeys.

The CV topography, as a function of site location was weak with only a few small but statistically significant trends. For $M 1$, the CV's orientation also varies as a function of corresponding site location on the medial-lateral $(\mathrm{m}-\mathrm{l})$ axis $\left[r_{\text {vertical } / \mathrm{m}-1}(85)=\right.$ -0.275 with $P=0.01]$ such that the more medial sites evoked movements with a larger horizontal component. M2 data (Fig. $1 C$ ), allowed for a larger sample of downward and upward movements. It suggests that the CV's orientation varies as a function of corresponding site location on the anterior-posterior (a-p) axis $\left[r_{\text {vertical/a-p }}(194)=0.507\right.$, with $\left.P \approx 10^{-14}\right]$ such that the CVs have a larger vertical component when they are evoked from more posterior sites. Moreover CV's direction varies as a function of site location on the $\mathrm{m}-1$ axis $\left[r_{\text {vertical } / \mathrm{m}-\mathrm{l}}(194)=-0.231\right.$, with $P=0.001]$ such that more medial sites evoked movements with larger horizontal component, similar to M1. Furthermore, deeper sites evoke more downward movements and more superficial sites 
evoke more upward movements $\left[r_{\text {vertical/depth }}(194)=-0.289\right.$, with $P \approx 10^{-5}$.

FRAMES OF REFERENCE. What frame of reference best describes the goal of the stimulation-evoked gaze shifts obtained in our experiments? Figure 5, left, shows simulated models of gaze trajectories, corresponding to three possibilities plotted in their correspondent coordinate systems. For instance, the goal could be $60^{\circ}$ upward of where the eye is pointing - a coordinate system fixed with the eye and plotted in an eye-fixed reference frame (Fig. $5 G$ ); or it could be $60^{\circ}$ upward of where the head is pointing, with a coordinate system fixed to the head and plotted in head-fixed reference frame (Fig. 5D); or $60^{\circ}$ upward of where the body is pointing, with a coordinate frame fixed to the body and plotted in body-fixed reference frame (Fig. 5A). When data are plotted in the intrinsic frame of the model, the simulated trajectories converge to a common point (Martinez-Trujillo et al. 2004).

There is also a fourth model, the fixed vector model (not simulated here), in which the simulated gaze movements are parallel with each other when plotted in space-fixed coordinates. For very small evoked saccades, this model is hard to distinguish from the eye-fixed model, but as movement size increases, the two models begin to diverge more and more (Crawford and Guitton 1997a; Klier et al. 2001). The different predictions of these two models will be addressed quantitatively in the following text.
We do not yet know the correct intrinsic frame for our data, but by corollary with the simulations, the actual stimulusevoked trajectories should converge best when plotted in this (intrinsic) frame such that if the LIP encodes a gaze motor command using one of the three reference frames (eye, head, or space), consequently the stimulation-evoked gaze shifts trajectories should converge when plotted in that reference frame.

To compare the stimulation data with the simulations, we selected real (stimulation-evoked) gaze shifts evoked from a variety of initial eye and head orientations (see METHODS), and we rotated them into three different coordinate systems (eye, head, and body) as we did with the simulated trajectories. Note that the data are plotted in three different frames, such that the frames used to plot the data correspond to the frames used for the simulated data. Figure 5 (middle and right) illustrates the gaze shifts trajectories evoked from stimulating two putative LIP sites-site 1 from $M 1$ and site 2 from $M 2$; the trajectories are plotted in three coordinate systems-space/body (1st row), head (2nd row), and eye (3rd row). For both monkeys, the trajectories appear to converge best when they are plotted in the eye-fixed coordinate system (Fig. 5, $H$ and $I$ ). We repeated the same visual analysis for all the stimulation LIP sites and made similar observations for every single site: qualitatively, the evoked gaze shifts most resembled the predictions of an eye-fixed (or fixed-vector) model. These observations, and the distinction between the latter two models, are quantified in the next two sections.
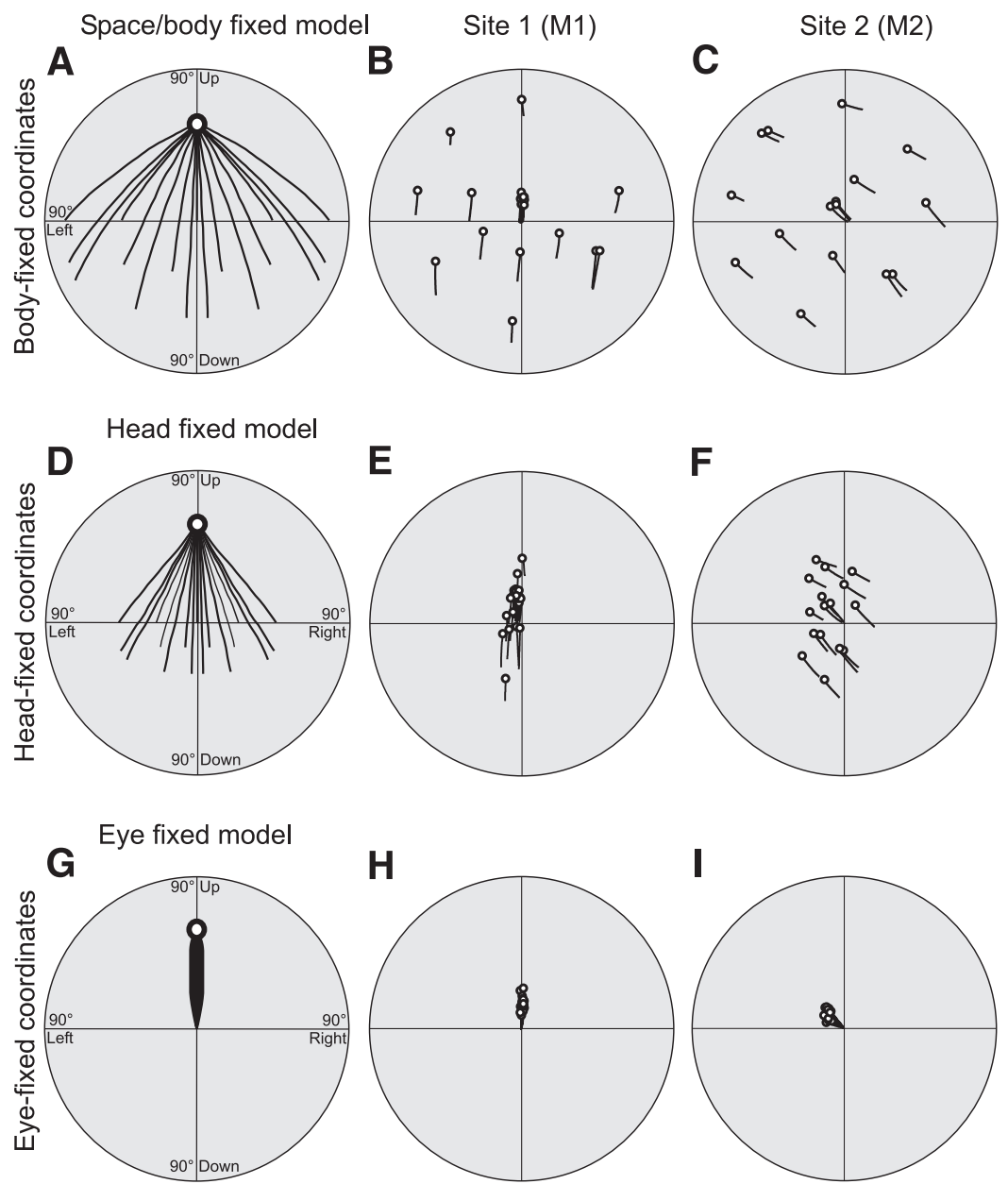

FIG. 5. Gaze trajectories plotted in different reference frames. Left: trajectories of movements simulated by 3 different models (after Martinez-Trujillo et al. 2004). The space/body model $(A)$ simulates movements that converge when they are plotted in body/space-fixed coordinate system. The head-fixed model $(D)$ simulates movements that converge when are plotted in head-fixed coordinate system; the eye-fixed model $(G)$ simulates movements that converge when plotted in eye-fixed coordinate system. Middle and right: trajectories of the gaze shifts evoked by stimulating 2 different sites (site 1 from $M 1$ and site 2 from M2). These trajectories are plotted into the 3 different coordinate systems: body-fixed $(B$ and $C$ ), head-fixed $(E$ and $F$ ), and eye-fixed ( $H$ and $I)$. Each point corresponds with the tip of the unit-length vector (computed from quaternionssee METHODS) and represents position for gaze, with the horizontal and vertical components projected from behind, using a linear scale. $\bigcirc$, end of the movement. 

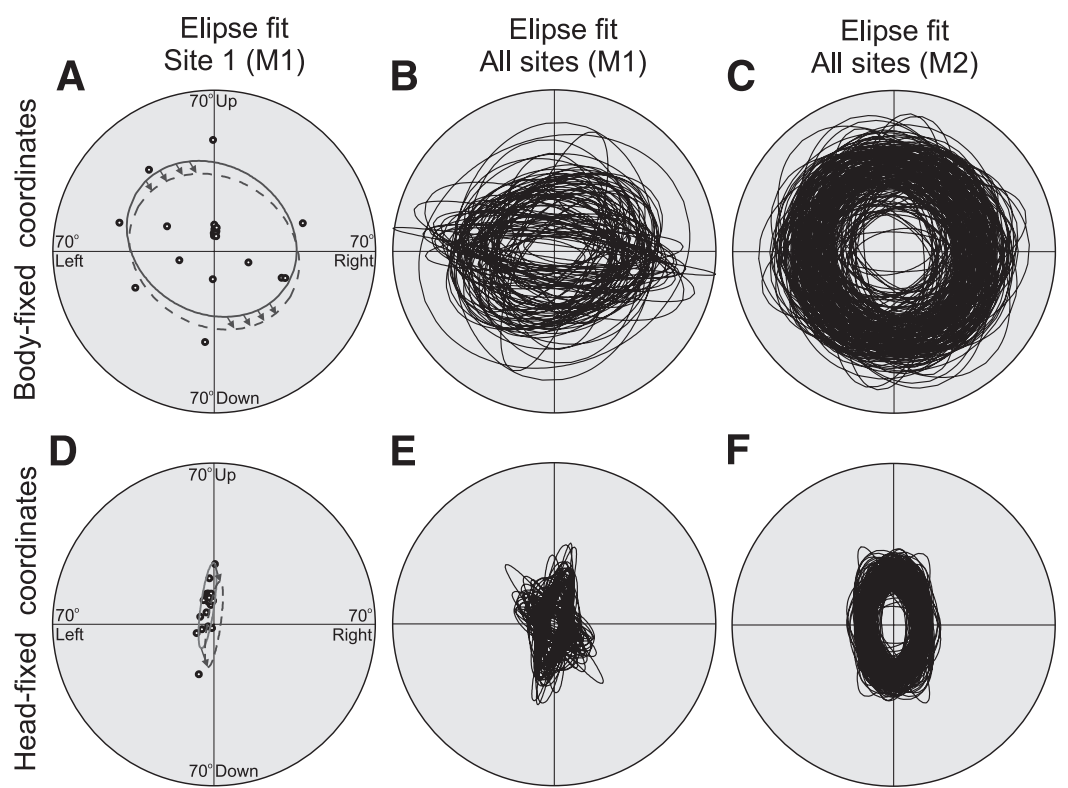

FIG. 6. Ellipse fits. Left: stimulation-evoked movement end positions (O) and the ellipse fitted for site 1 for the points plotted into body-fixed $(A)$, head-fixed $(D)$, and eye-fixed $(G)$ coordinate systems; plotted on a linear scale. - --, normalized ellipse-the center of fitted ellipse was translated onto the centers of the coordinate systems. $B, E$, and $H$ : Normalized ellipses fitted to all 85 sites from $M 1$ plotted into the 3 different reference frames. $C, F$, and $I$ : Normalized ellipses fitted to all 194 sites from $M 2$ plotted into 3 different reference frames.

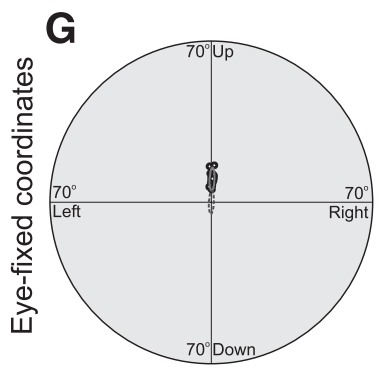

H

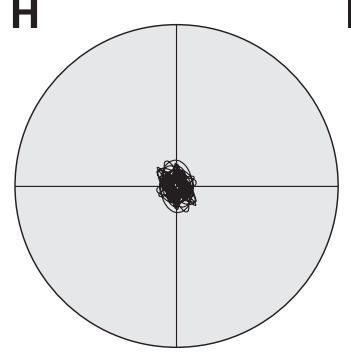

I

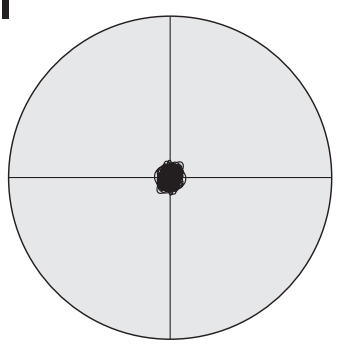

ELLIPSE FITTING. Following Martinez-Trujillo et al. (2004), we first utilized a simple method to quantify our data, focusing on the final positions of the stimulation-evoked gaze shifts plotted in different coordinate systems: space, head, and eye. Therefore we computed where the gaze was at the end of the stimulation-evoked movement and quantified the spread area for the endpoints gaze positions by fitting an ellipse to the cluster of points (see METHODS) in each reference frame and by comparing in which reference frame the ellipse's areas are the smallest.

Figure 6, left, an example of the ellipse fitting method. Each $O$ shows the end position of the stimulation-evoked gaze shifts, plotted in space (Fig. 6A), head (Fig. 6D), and eye (Fig. 6G) coordinate systems. The ellipse with the smallest area corresponds to data plotted in an eye reference frame (Fig. $6 G$ ). For an easier visual comparison, we realigned the fitted ellipses with the centers of the corresponding coordinate systems; the "new" normalized ellipse is plotted with a dotted line. The CVs in Fig. 3 show the approximate center of the gaze ellipses for all sites.

$B-I$ in Fig. 6 display the ellipses from all the sites included in the analysis for $M 1(B, E$, and $H)$ and $M 2(C, F$, and $I)$. For every site, the eye-fixed ellipses $(H$ and $I)$ had the smallest area (with an average value across sites of $31.86 \mathrm{deg}^{2}$ for $M 1$ and $22.34 \mathrm{deg}^{2}$ for M2), followed consecutively by the head-fixed ellipse ( $E$ and $F$; with an average value across sites of 231.35 $\mathrm{deg}^{2}$ for $M 1$ and $887.86 \mathrm{deg}^{2}$ for $M 2$ ), and finally the space/ body-fixed ellipse ( $B$ and $C$; with an average value across sites of 1,968.06 $\mathrm{deg}^{2}$ for $M 1$ and $4204.10 \mathrm{deg}^{2}$ for M2). The difference between the eye and head reference frames was statistically significant, with $P \approx 10^{-46}$ for $M 1$ and $P \approx 10^{-88}$ for $M 2$ (paired Student's $t$-test, after Bonferroni correction). The difference between the eye and space/body reference frames was also statistically significant with $P \approx 10^{-42}$ for $M 1$ and $P \approx 10^{-80}$ for $M 2$ (paired Student's $t$-test, after Bonferroni correction). Thus the ellipse areas are the smallest when they are fitted to data plotted in an eye reference frame for both monkeys. Because the large quantity of data in these plots tends to obscure many of the individual ellipses, we have supplied a graphical plot of the distributions of all the ellipse areas in each frame in supplementary Fig. $1 .{ }^{1}$ We found that the distributions of ellipse areas between sites was widest in space coordinates, intermediate in head coordinates, and far more narrow (i.e., consistent) in eye coordinates.

However, this method does not account for the possibility that the data may best fit an intermediate frame of reference. Moreover, it does not allow one to test between a truly fixed vector versus eye-fixed model, which was an important aspect of our previous analysis of gaze shifts evoked from the SC (Klier et al. 2001).

CI. This second, more sophisticated method of quantifying the convergence of the movements took into account the components of both the initial and final gaze position of the evoked movements. We computed convergence indices for direction and amplitude of the stimulation-evoked gaze shifts for each sites (see METHODS) and plotted them as a function of the CV direction and amplitude for that particular site. In the same plots, we generated predictions for the different models

\footnotetext{
${ }^{1}$ The online version of this article contains supplemental data.
} 
of reference frame coding and compared the data against such predictions. Thus the CI has a value of -1 for perfect convergence (movements are convergent to 1 point) and $\mathrm{CI}$ has a value of 0 if there is no convergence (the movements are parallel). For the eye-fixed model predictions, the convergence is also a function of the length of the movements (length of $\mathrm{CV})$. For smaller movements, the CI is close to zero and decreases exponentially for larger movements until reaching -1 for movements of $90^{\circ}$.

Figure 7, $A$ and $B$, shows the $\mathrm{CI}$ for the movement direction $\left(\mathrm{CI}_{\mathrm{Off}}\right.$ axis $)$ and movement amplitude $\left(\mathrm{CI}_{\mathrm{On} \text { axis }}\right)$ calculated for gaze in space/body-fixed reference frame for each site and plotted as a function of corresponding CV amplitude, for all sites, in both monkeys ( $M 1, \bigcirc$ and $M 2$, ๑). The space/bodyfixed model predicts a flat slope of $\mathrm{CI}=-1$, whereas the fixed-vector model predicts a flat slope of $\mathrm{CI}=0$. The - shows the predictions of an eye-fixed model for "off axis" data-data component orthogonal to the CV (Fig. 7A, $\rightarrow$ ), which is unfortunately very close to 0 (the fixed-vector model) in most of this range of movement (as calculated by Klier et al. 2001); too close to make a qualitative distinction between them based on visual inspection. However, these two predictions begin to diverge toward the right side of the panel (we will use this observation in the following text, to quantitatively test between these 2 models).

For the "on axis" data-component parallel to the CV (Fig. $7 B$ ), both the fixed-vector and the eye-fixed models predict values equal to 0 (gaze movements encoded by an eye-fixed model would be almost parallel when plotted in a space reference frame). A space/body-fixed model would predict perfect convergence, with CI values equal to -1 . Clearly, in
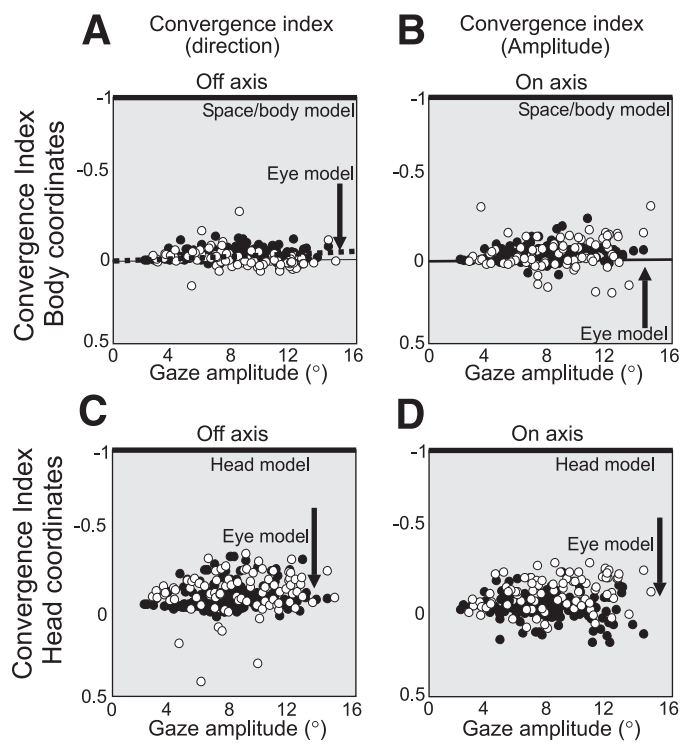

FIG. 7. Convergence indices. $A$ and $B$ : convergence indices (CIs) as a function of $\mathrm{CV}$ amplitude for $M 1(\mathrm{O})$ and for $M 2(\bullet)$ calculated for trajectories plotted in body/space-fixed coordinates, with the off axis component $(A)$ and the on axis component $(B)$. - , CI's values calculated by a simulated eye-fixed model (from Klier et al. 2001). A simulated space/body-fixed model would predict perfect convergence, with CI values of -1 . $C$ and $D$ : CIs as a function of the corresponding CV amplitude for $M 1(\circ)$ and for $M 2(\bullet)$, calculated for trajectories plotted in head-fixed coordinates, with the off axis component $(C)$ and the on axis component $(D)$. The simulated head-fixed model would predict perfect convergence, with CI values of -1 ; a simulated eye-fixed model would predict values closer to 0 . both panels (Fig. 7, $A$ and $B$ ), the data diverged widely from the predictions of the space/body-fixed model and agreed quite closely with the eye-fixed model. In both animals, these data were statistically different from the space model predictions $\left(P \approx 10^{-90}\right.$ for $M 1$ and $P \approx 10^{-200}$ for $M 2$; Student's $t$-test, after Bonferroni correction applied to both $\mathrm{CI}_{\text {Off axis }}$ and $\mathrm{CI}_{\mathrm{On} \text { axis }}$ ) with average values for $\mathrm{CI}_{\mathrm{Off}}$ axis of $-0.018 \pm 0.054$ for $M I$ and $-0.045 \pm 0.029$ for $M 2$ and average values for $\mathrm{CI}_{\mathrm{On}}$ axis $-0.038 \pm 0.041$ for $M 1$ and $-0.046 \pm 0.093$ for M2. The space/body-fixed model did not fit the population or even a single point.

However, for the CIs plotted in Fig. 7, top, the predictions of a head-fixed model cannot be represented by a single line because the predicted values depend on the relative contributions of the eyes and head to gaze, which can vary for different subjects or for different stimulation sites. In theory, the head- and eye-fixed models could make very similar predictions, depending on the animal's intrinsic patterns of eye-head coordination at the initial positions (MartinezTrujillo et al. 2004). Therefore to test if data follow the predictions of the head model, we calculated the CI for gaze movements rotated into a head reference frame. Figure 7, $C$ and $D$, plots the $\mathrm{CI}$ for the movement direction $\left(\mathrm{CI}_{\mathrm{Off}}\right.$ axis $)$ and movement amplitude $\left(\mathrm{CI}_{\mathrm{On} \text { axis }}\right)$ calculated for gaze in head-fixed reference frame and plotted as a function of corresponding CV amplitude for all sites, in both monkeys $(M 1, \bigcirc$ and $M 2, \bullet)$. In this case, the head model would predict values equal to -1 . The eye model does not predict specific values just that the values would be below the line of perfect convergence. Figure 7, $C$ and $D$, shows that in both animals, data were statistically different from the head-model predictions $\left(P \approx 10^{-70}\right.$ for $M 1$ and $P \approx 10^{-200}$ for $M 2$; Student's $t$-test, after Bonferroni correction applied to both $\mathrm{CI}_{\mathrm{Off} \text { axis }}$ and $\mathrm{CI}_{\text {On axis }}$ ) with average values for $\mathrm{CI}_{\text {Off axis }}$ of $-0.130 \pm 0.122$ for $M 1$ and $-0.096 \pm 0.069$ for $M 2$ and with average values for $\mathrm{CI}_{\mathrm{On} \text { axis }}$ of $-0.132 \pm 0.098$ for $M 1$ and $-0.050 \pm 0.068$ for $M 2$.

Thus in sharp contrast to similar data collected from the SEF (Martinez-Trujillo et al. 2004), we found no tendency here for the data to follow a distribution that fell between models and/or spanned across several models; the data simply followed an eye-fixed (or fixed-vector) curve. None of our LIP sites followed the predictions of either a space/body- or head-fixed model. However, for this range of gaze amplitudes (i.e., between 0 and $16^{\circ}$ ), there is no visible difference between the predictions of the eye-fixed and fixed-vector models in these plots. In fact, this difference only become clear for movements $>30^{\circ}$ and then becomes progressively larger for really big gaze shifts (Klier et al. 2001).

To summarize these results and test quantitatively between the fixed-vector and eye-centered models, we calculated the normalized squared residuals of the fits of the CI data to predictions of each model (between the data CIs and the CIs predicted by the 3 different models), following a method proposed by Martinez-Trujillo et al. (2004). The resulting values were plotted in Fig. 8, for M1 (top) and M2 (bottom), with residuals calculated for off and on axis CIs. The line graphs show that the residual values are smaller for the eye model predictions (data fits the eye model predictions) compared with the head or space model predictions. 

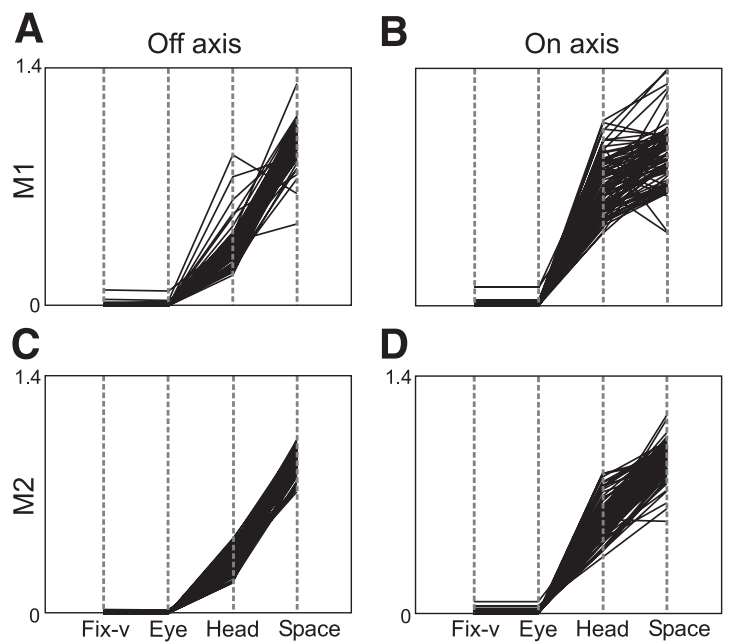

FIG. 8. Residuals plots. Each line represents one stimulation site and shows the residuals of fits to the data for the 4 different models: the fixed-vector (Fix-v), eye-fixed (Eye), head-fixed (Head), and space/body-fixed (Space) simulated models. The residuals were computed for the off axis CIs ( $A$ and $C$ ) and for the on axis CIs ( $B$ and $D$ ) for $M 1$ (top) and M2 (bottom).

Figure 8 re-emphasizes that the head- and space-fixed models are not even in the running. Moreover, here we had an opportunity to differentiate between the fixed-vector and eyefixed models. Although the difference was not clear graphically, there was a quantitative difference in the fits to these two models. On average across all sites $( \pm \mathrm{SD})$, the residual fit for the fixed vector model was $0.0031 \pm 0.0068, \sim 50 \%$ larger than the size of the average residuals for the eye-fixed model $0.0023 \pm 0.0061$. Pooling all data, there was a highly significant difference between these two fits $P \approx 10^{-17}$ (Student's $t$-test). Thus as was the case previously for the SC (but not for the SEFs), the eye-fixed model won out over the fixed-vector, head-fixed, and space/body-fixed models in our LIP data. Although this difference may not be important for the range of movements that we were able to LIP, it is perhaps useful to note that with this analysis, we have now eliminated the fixed-vector model from explaining the vast majority of our SC, LIP, FEF, and SEF data (Ascensio-Monteon et al. 2006; Constantin et al. 2004b; Klier et al. 2001; Martinez-Trujillo et al. 2004).

\section{Probability of evoking saccades as a function of gaze position}

For all of our stimulation sites, we observed qualitatively that stimulation did not evoke gaze movements on every trial. We further observed that on many stimulation sites, it seemed that the probability of evoking a movement when stimulated depended on where the monkey was looking (fixating a target) before we stimulated. The probability of evoking a movement for a given stimulation trial was not equal for all stimulation sites or for all initial target positions within a given stimulation site. In other words, the probability of evoking gaze shifts from different initial gaze position changed for each site of stimulation and for each target position within the stimulation site (Fig. 9, $A$ and $B$ ). The following section documents this effect quantitatively.

Figure 9 shows the process of calculating the probability of electrically eliciting saccadic eye movements, exemplified us- ing two stimulation sites. The first step (Fig. 9, $A$ and $B$ ) was counting the number of evoked gaze shifts (black traces) out of the total number of stimulations (gray and black dots) applied when the monkey was fixating on different LEDs. The gray and white arrows show the corresponding $\mathrm{CV}$ and $\mathrm{PV}$, respectively; the determination of the two vectors will be dealt with later (in Fig. 9, $E$ and $F$ ). The stimulation-evoked movements were selected only if the initial gaze orientation was located $\pm 10^{\circ}$ around the corresponding LED position; the 17 LEDs were being positioned in a 2-D space (horizontal-vertical plane) situated in front of the monkey. The resultant probability values (columns) for each LED area are plotted in Fig. 9, C
A
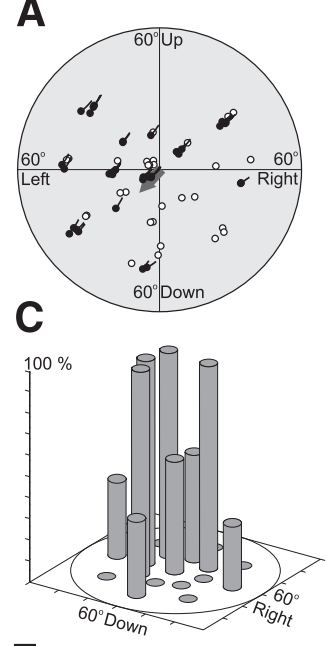

E

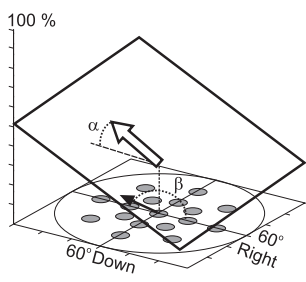

G

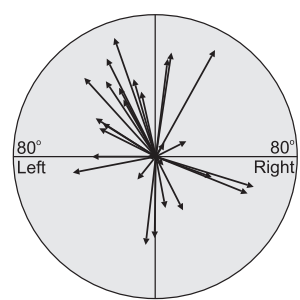

B
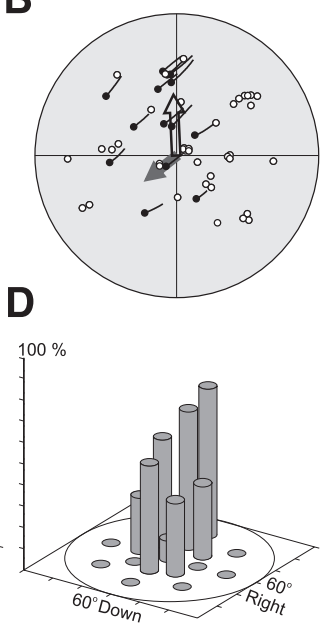

$\mathbf{F}$

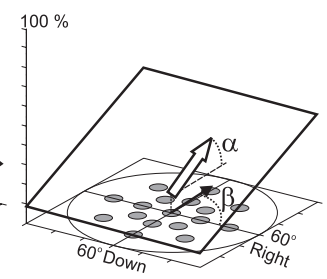

H

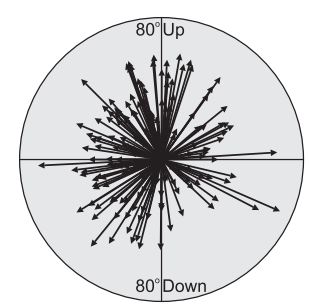

FIG. 9. Probability vectors (PVs). $A$ and $B$ : 1st step in calculating the probability of eliciting saccadic eye movements (exemplified with 2 different sites) is counting the number of stimulation-evoked gaze shifts (black trajectories) of the total number of stimulations (white and black circles). The movements were selected only if the initial gaze position was located $\pm 10^{\circ}$ around the LED. Outlined arrow, PV for that particular site; gray arrow, CV (CV) for that site. $C$ and $D$ : resultant probability columns corresponding to each of the 17 LEDs used in the experiment. $E$ and $F$ : 2 nd step was to fit a plane ("probability plane") to the resulting columns. The slope $(\alpha)$ and the orientation $(\beta)$ of the plane was expressed using a PV (outlined arrow). The amplitude of the PV was proportional to the amplitude of $\alpha$; the direction of the PV was proportional with $\beta$. $G$ and $H$ : we obtained $28 \mathrm{PVs}$ for $M 1(G)$ and 180 PVs for $M 2(H)$ with an increased variety of directions. There is no correlation between the PV (outlined arrow) and the corresponding CV (gray arrow) directions for any particular site (see $A$ and $B$ ). 
and $D$. The gaze shifts evoked from the stimulation sites illustrated in Fig. 9 showed that there is an increased probability of evoking movements when $M 1$ was fixating central and upper-left quadrant targets (Fig. $9 C$ ) and when $M 2$ was fixating central and upper quadrant targets (Fig. 9D). These probability graphs varied for different stimulation sites.

To quantify the directional vector for these gaze orientation effects, we fitted a plane (probability plane) to the resulting probability columns (Fig. 9, $E$ and $F$ ). These are planes fitted to the probability columns shown in $C$ and $D$ (see METHODs for details). We also tried higher-order fits, which of course made better fits, but it is unclear at this point whether these higherorder fits are justified without a much larger data set of stimulation trials from smaller position increments, needed to clearly distinguish between subtle signal and noise effects. Therefore the first-order plane fit used, allowed focus on the directional tuning of this response at the expense of possibly missing higher-order characteristics, such as localized hills and troughs.

Figure $9, E$ and $F$, graphically illustrates our complete analysis for the two representative stimulation sites used in the columns above. The slope $(\alpha)$ and orientation $(\beta)$ of the probability plane was expressed using a PV (outlined arrow in $E$ and $F$ ). The magnitude of $\mathrm{PV}$ was proportional with the tilt of the plane (the amplitude of $\alpha$ ) and the orientation of the PV was proportional with the angle between the fitted probability plane and the horizontal-vertical plane $(\beta)$. Applying the same analysis across the sites of stimulation, we obtained $28 \mathrm{PVs}$ for $M 1$ and 180 PVs for $M 2$ with an orientation that varied widely for both monkeys, covering the entire $360^{\circ}$ of the 2-D space.

The PVs for all sites of stimulation from animals $M 1$ and $M 2$ are illustrated in Fig. 9, $G$ and $H$, respectively. As one can see, the distribution of magnitude for these vectors (i.e., the tilt of the probability plane as a function of gaze position) was similar for both monkeys and quite prominent for most sites of stimulation-prominent enough that we could notice it qualitatively during experiments. However, there was no directional preference in these vectors (they covered the full range of directions). In other words, some stimulation sites could be more likely to evoke gaze shifts with the eyes looking up, others down, others lefts, others right, and anything in between. Moreover, we found no topographical correlation between the direction of these PVs and the anatomic location of the stimulation site-this organization appeared to be random.

We also investigated the possibility of any correlation between the PV and CV directions. For example, we wondered if the PV might align with the CV. For stimulation site 1 (Fig. $9 A$ ), the CV (gray thick, arrow) and the PV (white, thick arrow) are almost perpendicular to each other; for stimulation site 2 (Fig. 9B), the CV and PV have almost opposite directions, which seems to indicate that there is no correlation between the direction of the PV and the direction of the corresponding CV for these two particular sites of stimulation. Furthermore, across stimulation sites, there is no correlation between the probability of electrically eliciting saccadic eye movements and the average direction of the corresponding evoked gaze shifts $\left(R^{2}=0.031\right.$ for $M 1$ and $R^{2}=0.0004$ for $\left.M 2\right)$. In other words, for a given stimulation site with a given $\mathrm{CV}$, the PV could be in any direction.

\section{I S C U S S I O N}

The main result of our study was that gaze shifts evoked during stimulation of monkey LIP are encoded into an eyefixed reference frame. The second observation was that the probability of evoking these gaze shifts varied for each site as a function of initial gaze orientation.

\section{What is the frame of reference for LIP?}

Most previous single-unit recording studies of visual receptive fields in LIP have suggested an eye-fixed organization (Andersen et al. 1992; Boussaoud and Bremmer 1999; Colby et al. 1995; Culham et al. 2006). Many of these studies have also pointed out that these same neurons carry position information-in the form of gain fields-that would allow these neurons to implicitly encode higher-order frames of reference (Andersen et al. 1990b; Barash et al. 1991; Brotchie et al. 1995; Snyder et al. 1998). However, this is a topic that we will take up in a later section in the following text. In addition, the amplitude and direction of a given LIP unit's preferred saccade motor tuning usually overlaps with the location of the unit's eye-fixed visual receptive field (Andersen et al. 1987; Barash et al. 1991; Colby 1998; Colby et al. 1996; Wurtz et al. 2001). An exception to the general rule is a recent study, which suggests that LIP units may have eye-fixed, head-fixed, or perhaps body-fixed visual receptive fields (O'dhaniel et al. 2005).

Our stimulation data are generally consistent with the idea of an eye-fixed coding scheme in LIP. Our data clearly show that head- and body-fixed codes do not play as prominent a role in the output of the LIP, for example, as compared with that of the SEF (Martinez-Trujillo et al. 2004). It is possible that these signals play a more subtle role at the level of individual units but are simply swamped in the population vote cast during electrical stimulation. Moreover, our data also tend to rule out the fixed-vector model for LIP at least at a quantitative level. The difference between this model and the eye-fixed model is admittedly very small within the range that we tested here, but it is notable that the fixed-vector model has failed to provide a best fit for all but a very few sites in every area that we have tested to date. Moreover, an eye-centered code provides a common language for the LIP to communicate with other brain areas, such as the SC, where the difference between the two models is much more clear (Klier et al. 2001).

However, before drawing any conclusions, it is important to note that unit recording and stimulation are different techniques that show different things. This is not just a matter of unit recording showing the physiological activity of single neurons, as opposed the ensemble activation of a region of neurons and fibers (and their various orthodromic and antidromic projections) through stimulation. Receptive field mapping reveals correlations to the unit's inputs, whereas stimulation reveals the influence of an area's output on other regions of the brain (Pare and Wurtz 1997; Tehovnik et al. 2003). For example, when the equivalent experiments are simulated in artificial neural networks that implement a reference frame transformation, the receptive field mapping correlates with the reference frame of the input structures, whereas stimulation reveals the reference frame code of the output targets (Smith and Crawford 2005). Stimulation results may correlate better with the motor tuning of neurons yet even these two measures do not necessarily reveal the same thing in complex population 
coding schemes (Blohm et al. 2006). Thus it may be an oversimplification to think of LIP, or even single units within LIP, as using a single frame of reference to perform its computational role in the gaze control system.

Thus the best interpretation of our main result is that LIP projects to (and influences gaze behavior through) structures that in turn use an eye-fixed frame to code gaze. This is consistent with the known projections of the LIP to the FEFs and the SC, which are thought to code gaze in eye-centered coordinates (Klier et al. 2001; Russo and Bruce 1996; Stanton et al. 1995; Wurtz et al. 2001). Because most LIP units appear to have eye-fixed visual receptive fields (Ben Hamed and Duhamel 2002; Ben Hamed et al. 2001; Colby et al. 1996), this suggests that LIP is not primarily involved in transforming eye-fixed frame signals into a different frame (Smith and Crawford 2005). However, it does not rule out the existence of nonretinal receptive fields in LIP units (O'dhaniel et al. 2005). In our scheme, such neurons would be involved in a transformation from nonretinal frames (perhaps influenced by nonvisual projections to LIP such as auditory and somatosensory inputs) into an eye-centered frame.

Previous stimulation studies of LIP have not always shown such consistently eye-fixed coding. For example Thier and Andersen $(1996,1998)$ explored sites in which the movement vectors were either slightly or strongly modulated by eye position. The majority of sites with slight modulations are likely consistent with our eye-fixed results because this is what an eye-fixed scheme looks like when projected into space coordinates (Crawford and Guitton 1997b; Klier et al. 2001; Martinez-Trujillo et al. 2004). However, Thier and Andersen also described a group of sites, primarily in an "intercalated zone" located on the floor of the IPS sulcus that gave stronger position-dependent convergent movements and larger head movements than we observed in our study. We looked for a region like this but simply did not find it.

There could be several explanations for the difference between our results. First, it could be a difference in stimulation parameters. Our trains were longer, compared with those of Thier and Andersen $(1996,1998)$, but because the movements occurred early in the train, this does not seem to be an important difference. Second, Thier and Andersen occasionally used higher stimulus amplitudes $(\leq 400 \mu \mathrm{A})$, but this cannot account for the difference in the majority of our data, which used similar stimulus amplitudes. The most likely difference is that they used $500 \mathrm{~Hz}$, whereas we used a lower frequency $(300 \mathrm{~Hz})$. It is also possible that the behavioral set in our monkeys was different due to either previous training or experimental conditions during the stimulation experiment. For example, Thier and Andersen's monkeys' heads were partially restrained, whereas our monkeys' heads were completely unrestrained. Previous studies have shown that restraining the head produces much more convergent looking movements, especially in areas that produce larger eye + head gaze shifts when the head is not restrained (Freedman et al. 1996; Martinez-Trujillo et al. 2003a; Pare and Guitton 1990). Moreover, "freeing" the head completely may have further raised the stimulation threshold, causing us to miss this area at our stimulation parameters. Finally, although we tried to fully explore the area within and surrounding LIP, it is possible that we somehow missed the more convergent area described by Thier and Andersen (1996, 1998). However-assuming that we did not outright miss this area-it would appear that for one reason or another it may have a higher threshold for stimulation-evoked than LIP proper, perhaps placing it even further from the motor output than the main portion of LIP.

\section{Gaze position modulations in LIP}

Numerous studies have shown that activity in LIP neurons is modulated by eye position (as well as head position and vestibular information) in a way commonly referred to as a gain field (i.e., where location of the receptive field remains fixed in eye coordinates, but the sensitivity gain of the neuron is increased or decreased as a linear function of eye position) (Andersen et al. 1985, 1990b). It has been shown using neural network simulations that such gain fields can be used to rotate the preferred movement vector into different coordinate systems (Zipser and Andersen 1988). Normally one thinks in terms of using these signals to rotate eye-fixed information into other frames of reference for downstream motor structures; however, by the same token, this information could be used to rotate information from other sensory frames (such as auditory information in head coordinates) into an eye-fixed frame within LIP - consistent with a suggestion that we made in the preceding text-or to perform both functions. For example, although the primary outputs of LIP may be to structures such as the FEFs and the SC that seem to primarily use an eye-fixed code, LIP should also influence activity in the SEFs, which appears to employ a more complex coding scheme involving multiple reference frames at both the input and output levels (Martinez-Trujillo et al. 2004; Olson and Gettner 1999; Olson and Tremblay 2000; Park et al. 2006). It has also been shown that position information may also be necessary for other computations that involve LIP, such as the updating of targets in 3-D space during self-motion (Medendorp et al. 2002, 2003; Smith and Crawford 2001). For these reasons, gain fields are likely to be an important and useful feature within LIP.

It is not possible to test the gain fields of individual units using the microstimulation technique. It is not likely that the mass activation of many units through stimulation would emulate the normal function of gain fields, as gain fields can only perform reference frame transformations through the differential activation of different units with various motor tuning directions as a function of position (Smith and Crawford 2005). Although our stimulation-evoked movements followed an eye-fixed pattern that was not systematically influenced by initial gaze position (at least when the data are plotted in eye coordinates), they were strongly influenced in another way: the probability of evoking an eye movement from a given site depended on initial gaze position. Similar observations have been made for SC (Freedman et al. 1996) and SEF (MartinezTrujillo et al. 2003b).

It is possible that this result provides a causal signature for gain fields at the population level. In other words, if enough units in an area have gain fields that roughly align in their position-dependent tuning, this would increase the background firing rate for these units for gaze positions in this direction (Andersen et al. 1990b; Barash et al. 1991; Brotchie et al. 1995; Snyder et al. 1998), perhaps making it easier to evoke eye movements from these positions. This is not contradicted by our observation that our PVs did not correlate to our CVs (hypothetically corresponding to the 
position tuning of gain fields vs. the directional motor tuning of cells, respectively) across stimulation sites. We found the same random relationship between gain field tuning, visual tuning and motor tuning when an artificial neural network was trained to perform the 3-D reference frame transformation for saccades (Smith and Crawford 2005). In fact, the presence of nonorthogonalities between these elements is necessary to implement the nonlinear aspects of these transformations.

If these arguments are correct, then the PV provides a direct measure for mass coding, or perhaps even topography, of gain fields. However, this cannot be proven without a more direct comparison between these two phenomena; there could be many other reasons why the probability of a stimulationevoked eye movement from LIP is influenced by initial gaze position that have nothing to do with reference frame transformations, and/or these effects could have been mediated by downstream structures rather than within LIP.

\section{Role of LIP in gaze control}

Despite the fact that we were able to evoke gaze shifts from stimulation of a great many sites in the LIP region of each of our animals and despite the consistency of our results, two important caveats must be noted. First, higher current strengths were required to evoke gaze shifts from this area compared with other areas (FEFs, SEFs, and SC) where we otherwise used exactly the same stimulus parameters (Ascensio-Monteon et al. 2006; Klier et al. 2001; Martinez-Trujillo et al. 2003a,b ). Second, we did not evoke near the range and size of gaze shifts from LIP that we evoked from these other areas. The latter may arise from the lack of topography in LIP compared with areas like the SC, i.e., we may have been co-activating units that coded both large and small gaze shifts, leading to a vector-averaging effect (Gottlieb et al. 2005; Groh 2001; Thier and Andersen 1996, 1998). Nevertheless, these observations are consistent with the idea that LIP is not directly connected to the motor output of the gaze control system but rather is involved in more "cognitive" functions at the bridge between the sensory and motor stages of this system (Colby et al. 1996; Goldberg et al. 2002). What then is the place of the LIP within the reference frame transformations of this system?

LIP has strong and direct connections with the FEFs and the SC (Andersen et al. 1990a; Ferraina et al. 2002; Hanes and Wurtz 2001; Matelli and Luppino 2001; Pare and Wurtz 1997, 2001; Sparks et al. 2001; Stanton et al. 1988). This is supported by retrograde tracing studies confirming the anatomical connections from the LIP to the FEFs and SC and by electrical micro-stimulation studies confirming functional connections from the LIP to the FEFs and SC. According to these studies (Andersen et al. 1990a; Pare and Wurtz 1997; Schall et al. 1995), LIP has direct motor output to the SC-with strong connections to the intermediate layers of the SC. For this reason, it is convenient that LIP and the SC (and most sites in the FEF) appear to use a common eye-centered motor code. Our single-unit recording studies (DeSouza et al. 2006) and our microstimulation studies (Constantin et al. 2004a; Klier et al. 2001) in the head-unrestrained monkey suggest that the SC integrates its inputs into a primarily eye-fixed motor code for gaze commands. This in turn suggests that the nonlinear transformation of this signal into a motor code for eye and head muscles, which is highly significant in the head-free range, is performed at stages even later than the SC, perhaps at the level of the last few premotor synapses of the serial projection (Klier et al. 2001).

However, LIP is also thought to have some projections to the SEFs (Huerta and Kaas 1990; Luppino and Rizzolatti 2000; Matelli and Luppino 2001). As mentioned in the preceding text, this area appears to use a much more complex code that shows both ego- and object-centered signals at the level of individual units (Olson and Tremblay 2000) and multiple egocentric reference frames (eye, head, and space/body) at the level of stimulus-evoked gaze shifts (Martinez-Trujillo et al. 2004; Park et al. 2006; Schall et al. 1995). The SEFs in turn project to the FEFs (Bruce et al. 1985; Luppino and Rizzolatti 2000; Schall et al. 1995; Stanton et al. 1988). In our recent head-free stimulation study of the FEF (where we used the same paradigm as in the current study), we found that most sites used an eye-fixed code, but some (perhaps targets of the $\mathrm{SEF}$ ) used a more head-fixed code (Ascensio-Monteon et al. 2006). In this regard, this placed the FEF in a position intermediate between the SEF and SC.

These observations and the data within our current study are consistent with our recent suggestion that there are two cortical streams within the higher levels of the gaze control system (Martinez-Trujillo et al. 2004). One pathway-running directly from visual cortex through LIP to the SC, and indirectly through LIP to the SC via FEF-that uses a primarily eye-centered code to affect geometrically simple aspects of the visuomotor aspects of gaze. This pathway would include and be an elaboration of the phylogenetically older subcortical paths directly from the eye to the optic tectum (Kostyk and Grobstein 1987a,b; Masino and Grobstein 1989), where now LIP interposes between the visual and motor layers of the SC (Albano et al. 1982; Wurtz and Albano 1980). A second pathway-running through LIP to the SEF to FEF and perhaps other more high level structures-is used for more complex spatial aspects of gaze control. This series of nested loops could provide the primate-compared with the amphibian, for example-with the capacity for reflexive gaze shifts, spatially simple gaze shifts with cognitive transformations within an eye-centered frame, and gaze shifts that involve more complex objectcentered and body-centered plans. This scheme places LIP with an important early role within both of the latter two cognitive streams of computation.

\section{REFEREN CES}

Ascencio-Monteon J, Wang H, Martinez-Trujillo JC, Crawford JD. Frames of reference for eye-head gaze shifts evoked during stimulation of the primate frontal eye fields. Soc Neurosci Abstr 32: 139.7, 2006.

Albano JE, Mishkin M, Westbrook LE, Wurtz RH. Visuomotor deficits following ablation of monkey superior colliculus. J Neurophysiol 48: 338 351, 1982.

Andersen RA. Visual and eye movement functions of the posterior parietal cortex. Annu Rev Neurosci 12: 377-403, 1989.

Andersen RA, Asanuma C, Essick G, Siegel RM. Corticocortical connections of anatomically and physiologically defined subdivisions within the inferior parietal lobule. J Comp Neurol 296: 65-113, 1990a.

Andersen RA, Bracewell RM, Barash S, Gnadt JW, Fogassi L. Eye position effects on visual, memory, and saccade-related activity in areas LIP and 7a of macaque. J Neurosci 10: 1176-1196, 1990b.

Andersen RA, Brotchie PR, Mazzoni P. Evidence for the lateral intraparietal area as the parietal eye field. Curr Opin Neurobiol 2: 840-846, 1992. 
Andersen RA, Buneo CA. Intentional maps in posterior parietal cortex. Annu Rev Neurosci 25: 189-220, 2002.

Andersen RA, Essick GK, Siegel RM. Encoding of spatial location by posterior parietal neurons. Science 230: 456-458, 1985.

Andersen RA, Essick GK, Siegel RM. Neurons of area 7 activated by both visual stimuli and oculomotor behavior. Exp Brain Res 67: 316-322, 1987.

Andersen RA, Snyder LH, Batista AP, Buneo CA, Cohen YE. Posterior parietal areas specialized for eye movements (LIP) and reach (PRR) using a common coordinate frame. Novartis Found Symp 218: 109-122, 1998.

Andersen RA, Snyder LH, Bradley DC, Xing J. Multimodal representation of space in the posterior parietal cortex and its use in planning movements. Annu Rev Neurosci 20: 303-330, 1997.

Andersen RA, Zipser D. The role of the posterior parietal cortex in coordinate transformations for visual-motor integration. Can J Physiol Pharmacol 66: 488-501, 1988 .

Astafiev SV, Shulman GL, Stanley CM, Snyder AZ, Van Essen DC, Corbetta M. Functional organization of human intraparietal and frontal cortex for attending, looking, and pointing. J Neurosci 23: 4689-4699, 2003.

Barash S, Bracewell RM, Fogassi L, Gnadt JW, Andersen RA. Saccaderelated activity in the lateral intraparietal area. II. Spatial properties. J Neurophysiol 66: 1109-1124, 1991.

Ben Hamed S, Duhamel JR. Ocular fixation and visual activity in the monkey lateral intraparietal area. Exp Brain Res 142: 512-528, 2002.

Ben Hamed S, Duhamel JR, Bremmer F, Graf W. Representation of the visual field in the lateral intraparietal area of macaque monkeys: a quantitative receptive field analysis. Exp Brain Res 140: 127-144, 2001.

Bizzi E, Polit A, Morasso P. Mechanisms underlying achievement of final head position. J Neurophysiol 39: 435-444, 1976.

Blohm G, Keith GP, Crawford JC. A possible neural basis of the 3D reference frame transformation for reaching. Soc Neurosci Abstr 32: 307.6, 2006.

Boussaoud D, Bremmer F. Gaze effects in the cerebral cortex: reference frames for space coding and action. Exp Brain Res 128: 170-180, 1999.

Brotchie PR, Andersen RA, Snyder LH, Goodman SJ. Head position signals used by parietal neurons to encode locations of visual stimuli. Nature 375: 232-235, 1995.

Bruce CJ, Goldberg ME, Bushnell MC, Stanton GB. Primate frontal eye fields. II. Physiological and anatomical correlates of electrically evoked eye movements. J Neurophysiol 54: 714-734, 1985.

Chen LL, Walton MM. Head movement evoked by electrical stimulation in the supplementary eye field of the rhesus monkey. J Neurophysiol 94: 4502-4519, 2005.

Cohen YE, Andersen RA. A common reference frame for movement plans in the posterior parietal cortex. Nat Rev Neurosci 3: 553-562, 2002.

Colby CL. Action-oriented spatial reference frames in cortex. Neuron 20: 15-24, 1998.

Colby CL, Duhamel JR. Spatial representations for action in parietal cortex. Brain Res Cogn Brain Res 5: 105-115, 1996.

Colby CL, Duhamel JR, Goldberg ME. Oculocentric spatial representation in parietal cortex. Cereb Cortex 5: 470-481, 1995.

Colby CL, Duhamel JR, Goldberg ME. Visual, presaccadic, and cognitive activation of single neurons in monkey lateral intraparietal area. $J$ Neurophysiol 76: 2841-2852, 1996.

Constantin AG, Wang H, Crawford JD. Role of superior colliculus in adaptive eye-head coordination during gaze shifts. J Neurophysiol 92: 2168-2184, 2004a.

Constantin AG, Wang H, Martinez JC and Crawford JD. Properties of gaze shifts evoked by stimulating lateral intraparietal cortex (LIP) in the head-unrestrained monkey. Soc Neurosci Abstr 30: 186.8, $2004 \mathrm{~b}$.

Corneil BD, Olivier E, Munoz DP. Neck muscle responses to stimulation of monkey superior colliculus. II. Gaze shift initiation and volitional head movements. J Neurophysiol 88: 2000-2018, 2002.

Crawford JD, Ceylan MZ, Klier EM, Guitton D. Three-dimensional eyehead coordination during gaze saccades in the primate. J Neurophysiol 81: 1760-1782, 1999.

Crawford JD, Guitton D. Primate head-free saccade generator implements a desired (post-VOR) eye position command by anticipating intended head motion. J Neurophysiol 78: 2811-2816, 1997a.

Crawford JD, Guitton D. Visual-motor transformations required for accurate and kinematically correct saccades. J Neurophysiol 78: 1447-1467, 1997b.

Culham JC, Cavina-Pratesi C, Singhal A. The role of parietal cortex in visuomotor control: what have we learned from neuroimaging? Neuropsychologia 44: 2668-2684, 2006.
Cutrell EB, Marrocco RT. Electrical microstimulation of primate posterior parietal cortex initiates orienting and alerting components of covert attention. Exp Brain Res 144: 103-113, 2002.

Desouza JF, Yan X, Blohm G, Wang H, Crawford JD. Gaze position effects and position-dependent motor tuning in primate superior colliculus (SC) neurons during head-unrestrained visually guided movements. Soc Neurosci Abstr 32: 211.1, 2006.

Dominey PF, Schlag J, Schlag-Rey M, Arbib MA. Colliding saccades evoked by frontal eye field stimulation: artifact or evidence for an oculomotor compensatory mechanism underlying double-step saccades? Biol Cybern 76: 41-52, 1997.

Ferraina S, Pare M, Wurtz RH. Comparison of cortico-cortical and corticocollicular signals for the generation of saccadic eye movements. J Neurophysiol 87: 845-858, 2002.

Freedman EG, Stanford TR, Sparks DL. Combined eye-head gaze shifts produced by electrical stimulation of the superior colliculus in rhesus monkeys. J Neurophysiol 76: 927-952, 1996.

Gaymard B, Lynch J, Ploner CJ, Condy C, Rivaud-Pechoux S. The parieto-collicular pathway: anatomical location and contribution to saccade generation. Eur J Neurosci 17: 1518-1526, 2003.

Glenn B, Vilis T. Violations of Listing's law after large eye and head gaze shifts. J Neurophysiol 68: 309-318, 1992.

Goldberg ME, Bisley J, Powell KD, Gottlieb J, Kusunoki M. The role of the lateral intraparietal area of the monkey in the generation of saccades and visuospatial attention. Ann NY Acad Sci 956: 205-215, 2002.

Gottlieb J, Kusunoki M, Goldberg ME. Simultaneous representation of saccade targets and visual onsets in monkey lateral intraparietal area. Cereb Cortex 15: 1198-1206, 2005.

Graziano MS, Gross CG. Spatial maps for the control of movement. Curr Opin Neurobiol 8: 195-201, 1998.

Groh JM. Converting neural signals from place codes to rate codes. Biol Cybern 85: 159-165, 2001.

Guitton D, Volle M. Gaze control in humans: eye-head coordination during orienting movements to targets within and beyond the oculomotor range. J Neurophysiol 58: 427-459, 1987.

Hanes DP, Patterson WF 2nd, Schall JD. Role of frontal eye fields in countermanding saccades: visual, movement, and fixation activity. $J$ Neurophysiol 79: 817-834, 1998.

Hanes DP, Wurtz RH. Interaction of the frontal eye field and superior colliculus for saccade generation. J Neurophysiol 85: 804-815, 2001.

Hanks TD, Ditterich J, Shadlen MN. Microstimulation of macaque area LIP affects decision-making in a motion discrimination task. Nat Neurosci 9: 682-689, 2006

Huerta MF, Kaas JH. Supplementary eye field as defined by intracortical microstimulation: connections in macaques. J. comp. neurol. 293: 299-330, 1990.

Ipata AE, Gee AL, Goldberg ME, Bisley JW. Activity in the lateral intraparietal area predicts the goal and latency of saccades in a free-viewing visual search task. J Neurosci 26: 3656-3661, 2006.

Klier EM, Crawford JD. Neural control of three-dimensional eye and head posture. Ann NY Acad Sci 1004: 122-131, 2003.

Klier EM, Martinez-Trujillo JC, Medendorp WP, Smith MA, Crawford JD. Neural control of 3-D gaze shifts in the primate. Prog Brain Res 142: $109-124,2003$.

Klier EM, Wang H, Crawford JD. Neural mechanisms of three-dimensional eye and head movements. Ann NY Acad Sci 956: 512-514, 2002.

Klier EM, Wang H, Crawford JD. The superior colliculus encodes gaze commands in retinal coordinates. Nat Neurosci 4: 627-632, 2001.

Kostyk SK, Grobstein P. Neuronal organization underlying visually elicited prey orienting in the frog. II. Anatomical studies on the laterality of central projections. Neuroscience 21: 57-82, 1987a.

Kostyk SK, Grobstein P. Neuronal organization underlying visually elicited prey orienting in the frog. III. Evidence for the existence of an uncrossed descending tectofugal pathway. Neuroscience 21: 83-96, 1987b.

Kurylo DD, Skavenski AA. Eye movements elicited by electrical stimulation of area PG in the monkey. J Neurophysiol 65: 1243-1253, 1991.

Li CS, Andersen RA. Inactivation of macaque lateral intraparietal area delays initiation of the second saccade predominantly from contralesional eye positions in a double-saccade task. Exp Brain Res 137: 45-57, 2001.

Linden JF, Grunewald A, Andersen RA. Responses to auditory stimuli in macaque lateral intraparietal area. II. Behavioral modulation. J Neurophysiol 82: 343-358, 1999

Luppino G, Rizzolatti G. The organization of the frontal motor cortex. News Physiol Sci 15: 219-224, 2000. 
Martinez-Trujillo JC, Klier EM, Wang H, Crawford JD. Contribution of head movement to gaze command coding in monkey frontal cortex and superior colliculus. J Neurophysiol 90: 2770-2776, 2003a.

Martinez-Trujillo JC, Medendorp WP, Wang H, Crawford JD. Frames of reference for eye-head gaze commands in primate supplementary eye fields. Neuron 44: 1057-1066, 2004.

Martinez-Trujillo JC, Wang H, Crawford JD. Electrical stimulation of the supplementary eye fields in the head-free macaque evokes kinematically normal gaze shifts. J Neurophysiol 89: 2961-2974, 2003b.

Masino T, Grobstein P. The organization of descending tectofugal pathways underlying orienting in the frog, Rana pipiens. II. Evidence for the involvement of a tecto-tegmento-spinal pathway. Exp Brain Res 75: 245-264, 1989.

Matelli M, Luppino G. Parietofrontal circuits for action and space perception in the macaque monkey. Neuroimage 14: S27-32, 2001.

Mazzoni P, Bracewell RM, Barash S, Andersen RA. Motor intention activity in the macaque's lateral intraparietal area. I. Dissociation of motor plan from sensory memory. J Neurophysiol 76: 1439-1456, 1996.

Medendorp WP, Goltz HC, Vilis T, Crawford JD. Gaze-centered updating of visual space in human parietal cortex. J Neurosci 23: 6209-6214, 2003.

Medendorp WP, Smith MA, Tweed DB, Crawford JD. Rotational remapping in human spatial memory during eye and head motion. J Neurosci 22 : RC196, 2002

Mushiake H, Fujii N, Tanji J. Microstimulation of the lateral wall of the intraparietal sulcus compared with the frontal eye field during oculomotor tasks. J Neurophysiol 81: 1443-1448, 1999.

O'dhaniel A, Mullette-Gillman OA, Cohen YE, Groh JM. Eye-centered, head-centered, and complex coding of visual and auditory targets in the intraparietal sulcus. J Neurophysiol 94: 2331-2352, 2005.

Olson CR, Gettner SN. Macaque SEF neurons encode object-centered directions of eye movements regardless of the visual attributes of instructional cues. J Neurophysiol 81: 2340-2346, 1999.

Olson CR, Tremblay L. Macaque supplementary eye field neurons encode object-centered locations relative to both continuous and discontinuous objects. J Neurophysiol 83: 2392-2411, 2000.

Pare M, Guitton D. Gaze-related activity of brainstem omnipause neurons during combined eye-head gaze shifts in the alert cat. Exp Brain Res 83: 210-214, 1990 .

Pare M, Wurtz RH. Monkey posterior parietal cortex neurons antidromically activated from superior colliculus. J Neurophysiol 78: 3493-3497, 1997.

Pare M, Wurtz RH. Progression in neuronal processing for saccadic eye movements from parietal cortex area lip to superior colliculus. J Neurophysiol 85: 2545-2562, 2001.

Park J, Schlag-Rey M, Schlag J. Frames of reference for saccadic command tested by saccade collision in the supplementary eye field. $J$ Neurophysiol 95: 159-170, 2006

Platt ML, Glimcher PW. Responses of intraparietal neurons to saccadic targets and visual distractors. J Neurophysiol 78: 1574-1589, 1997.

Roucoux A, Guitton D, Crommelinck M. Stimulation of the superior colliculus in the alert cat. II. Eye and head movements evoked when the head is unrestrained. Exp Brain Res 39: 75-85, 1980.

Russo GS, Bruce CJ. Effect of eye position within the orbit on electrically elicited saccadic eye movements: a comparison of the macaque monkey's frontal and supplementary eye fields. J Neurophysiol 69: 800-818, 1993.

Russo GS, Bruce CJ. Neurons in the supplementary eye field of rhesus monkeys code visual targets and saccadic eye movements in an oculocentric coordinate system. J Neurophysiol 76: 825-848, 1996.

Russo GS, Bruce CJ. Supplementary eye field: representation of saccades and relationship between neural response fields and elicited eye movements. J Neurophysiol 84: 2605-2621, 2000.

Schall JD. The neural selection and control of saccades by the frontal eye field. Philos Trans R Soc Lond B Biol Sci 357: 1073-1082, 2002.
Schall JD, Morel A, King DJ, Bullier J. Topography of visual cortex connections with frontal eye field in macaque: convergence and segregation of processing streams. J Neurosci 15: 4464-4487, 1995.

Schiller PH, Tehovnik EJ. Neural mechanisms underlying target selection with saccadic eye movements. Prog Brain Res 149: 157-171, 2005.

Schlag J, Schlag-Rey M. Colliding saccades may reveal the secret of their marching orders. Trends Neurosci 13: 410-415, 1990.

Shibutani H, Sakata H, Hyvarinen J. Saccade and blinking evoked by microstimulation of the posterior parietal association cortex of the monkey. Exp Brain Res 55: 1-8, 1984.

Smith MA, Crawford JD. Implications of ocular kinematics for the internal updating of visual space. J Neurophysiol 86: 2112-2117, 2001.

Smith MA, Crawford JD. Distributed population mechanism for the 3-D oculomotor reference frame transformation. J Neurophysiol 93: 1742-1761, 2005 .

Snyder LH, Grieve KL, Brotchie P, Andersen RA. Separate body- and world-referenced representations of visual space in parietal cortex. Nature 394: 887-891, 1998

Soechting JF, Flanders M. Moving in three-dimensional space: frames of reference, vectors, and coordinate systems. Annu Rev Neurosci 15: 167-191, 1992.

Sparks DL, Freedman EG, Chen LL, Gandhi NJ. Cortical and subcortical contributions to coordinated eye and head movements. Vision Res 41: 3295-3305, 2001.

Stanton GB, Bruce CJ, Goldberg ME. Topography of projections to posterior cortical areas from the macaque frontal eye fields. J Comp Neurol 353: 291-305, 1995

Stanton GB, Goldberg ME, Bruce CJ. Frontal eye field efferents in the macaque monkey. II. Topography of terminal fields in midbrain and pons. J Comp Neurol 271: 493-506, 1988.

Tehovnik EJ, Slocum WM, Schiller PH. Saccadic eye movements evoked by microstimulation of striate cortex. Eur J Neurosci 17: 870-878, 2003.

Tehovnik EJ, Sommer MA, Chou IH, Slocum WM, Schiller PH. Eye fields in the frontal lobes of primates. Brain Res Brain Res Rev 32: 413-448, 2000.

Thier P, Andersen RA. Electrical microstimulation suggests two different forms of representation of head-centered space in the intraparietal sulcus of rhesus monkeys. Proc Natl Acad Sci USA 93: 4962-4967, 1996.

Thier P, Andersen RA. Electrical microstimulation distinguishes distinct saccade-related areas in the posterior parietal cortex. J Neurophysiol 80: 1713-1735, 1998.

Tu TA, Keating EG. Electrical stimulation of the frontal eye field in a monkey produces combined eye and head movements. J Neurophysiol 84: 11031106, 2000

Tweed D, Cadera W, Vilis T. Computing three-dimensional eye position quaternions and eye velocity from search coil signals. Vision Res 30: 97-110, 1990.

Tweed D, Glenn B, Vilis T. Eye-head coordination during large gaze shifts. J Neurophysiol 73: 766-779, 1995.

Tweed D, Vilis T. Geometric relations of eye position and velocity vectors during saccades. Vision Res 30: 111-127, 1990.

Westheimer G. Kinematics of the eye. J Opt Soc Am 47: 967-974, 1957.

Wurtz RH, Albano JE. Visual-motor function of the primate superior colliculus. Annu Rev Neurosci 3: 189-226, 1980.

Wurtz RH, Sommer MA, Pare M, Ferraina S. Signal transformations from cerebral cortex to superior colliculus for the generation of saccades. Vision Res 41: 3399-3412, 2001.

Zipser D, Andersen RA. A back-propagation programmed network that simulates response properties of a subset of posterior parietal neurons. Nature 331: 679-684, 1988 